\title{
ARTÍCULO
}

\section{EL RETORNO ECONÓMICO DE LA EDUCACIÓN MEDIA TÉCNICO PROFESIONAL EN CHILE*}

\author{
Alonso Bucarey \\ MIT \\ Sergio Urzúa \\ University of Maryland y CEP
}

\begin{abstract}
Resumen: Este trabajo analiza el impacto de asistir a un establecimiento de educación media técnico profesional (TP) sobre el desempeño en el mercado laboral. Utilizando datos administrativos de salarios y la información contenida en la
\end{abstract}

Alonso BuCARey. Licenciado y magíster en Economía, Universidad de Chile. Actualmente es alumno del programa de doctorado en Economía en MIT (Mass.). Dirección electrónica: alonsobucarey@gmail.com.

Sergio Urzúa. Ph.D. en Economía de la Universidad de Chicago. Profesor del departamento de economía de la Universidad de Maryland y jefe del área de políticas sociales del Centro de Estudios Públicos. Dirección electrónica:surzua@cepchile.cl.

* Agradecemos los comentarios de Dante Contreras, Juan Echenique, Claudia Martínez, Esteban Puentes, Jaime Ruiz-Tagle y a dos árbitros anónimos de Estudios Públicos. Agradecemos la colaboración del Ministerio de Hacienda. Los autores no usaron información que les permitiera identificar a individuos particulares. Los datos fueron utilizados bajo estrictos protocolos de seguridad. Sergio Urzúa agradece el apoyo de la Iniciativa Científica Milenio del Ministerio de Economía, Proyecto NS10004. Alonso Bucarey agradece el apoyo financiero de CONICYT. 
prueba SIMCE, identificamos el tipo de colegio en que los individuos cursaron sus estudios secundarios (2001) y luego observamos los meses de empleo formal (asalariado) y salarios para el período 2007-2011.

Nuestros resultados muestran que la educación TP tiene efectos positivos sobre el número de meses de empleo formal. En particular, los efectos sobre empleo fluctúan entre 0,4 y 1,6 meses de empleo adicionales al año dependiendo de la categoría de educación TP considerada. Por otra parte, este tipo de educación tiene, en promedio, un efecto negativo sobre salarios. Sin embargo, esta conclusión oculta una importante heterogeneidad. La categoría de educación TP Industrial resulta siempre competitiva frente a la educación científicohumanista $(\mathrm{CH})$ con un efecto de 42.100 pesos mensuales adicionales al considerar el salario promedio de 12 meses. En contraste, quienes estudiaron en un colegio de tipo TP Técnico ganarían en promedio 54.610 pesos menos que sus pares de colegios $\mathrm{CH}$. En conjunto nuestros resultados muestran un escenario optimista en términos de empleo para los egresados de liceos TP, aunque la heterogeneidad que reportamos entre las categorías representa un serio desafío desde el punto de vista de políticas públicas.

Palabras clave: educación técnico-profesional, retorno de la educación, evaluación de políticas públicas.

Recibido: diciembre 2012; aceptado: marzo 2013.

\section{ECONOMIC RETURN TO VOCATIONAL SECONDARY EDUCATION IN CHILE}

Abstract: This paper analyzes the effect of attending vocational secondary education on labor market outcomes. Using administrative data on school enrollment, labor income, and employment, we identify the type of secondary school attended, as well as the number of months of formal employment and monthly income, ten years after graduating from high school. We supplement our data with historical records about the local availability of vocational secondary education, which we use to construct instrumental variables. Our results show that compared to conventional secondary schools, vocational secondary education increases formal employment - between 0.4 and 1.6 months of employment per year depending on its type. On the other hand, we document 
that graduates from vocational schools have, on average, lower wages than those of individuals with conventional high school degrees. However, we find significant heterogeneity by type of vocational education. For example, our results show that industry-oriented vocational schools over perform the other alternatives, including conventional secondary education. For this type, we estimate a return of 42,100 Chilean pesos per month. In contrast, students who attended technical high schools earn 54,610 Chilean pesos less than those obtaining high school degrees from conventional high schools.

Overall, our findings provide new insights on the return to vocational education in Chile. They inform policy makers about the challenges and potential benefits associated with non-conventional high schools.

Keywords: vocational secondary education, return to education, public policy evaluation..

Received: December 2012; accepted: March 2013.

\section{Introducción}

Q mentado cambios significativos. El porcentaje de estudiantes matriculados en establecimientos educacionales públicos pasó de 57,8\% en 1990 a menos del $47 \%$ en el 2012. Por otra parte, se ha producido un aumento sin precedente en la matrícula de la educación superior. En veinte años el número de matriculados en educación superior se quintuplicó y recientemente se ha discutido ampliamente la gran heterogeneidad en los retornos económicos asociados a los títulos profesionales ofrecidos en el sistema de educación superior ${ }^{1}$. A su vez, la demanda por una educa-

1 Urzúa (2012) muestra, utilizando datos de salarios promedios por carrera, que existen universidades que ofrecen títulos profesionales que entregan retornos menores a los que los individuos habrían obtenido en el evento (contra-factual) de ingresar al mercado laboral luego de terminar la educación secundaria. Reyes, Rodríguez y Urzúa (2012) encuentran el mismo resultado utilizando un modelo estructural. Estos resultados sugieren que la educación secundaria puede representar una alternativa relevante para un grupo importante de jóvenes chilenos, y por tanto la distinción entre educación media $\mathrm{CH}$ y TP debe ser explorada de mejor manera. 
ción de calidad, en todos los niveles, ha movilizado a estudiantes como también la agenda de políticas públicas en el sector. Sin embargo, la educación media y, en particular, la educación Técnico-Profesional (TP) ha estado largamente ausente del debate. En este trabajo presentamos la primera evidencia del efecto de este tipo de educación sobre el desempeño en el mercado laboral de sus egresados ${ }^{2}$.

El sistema de educación secundaria en Chile considera dos modalidades: la educación Científico-Humanista $(\mathrm{CH})$ y la educación TP. La educación media $\mathrm{CH}$ busca profundizar los contenidos de formación general, preparando a sus egresados para continuar sus estudios en el nivel de educación terciario. Por otra parte, la educación TP tiene como objetivo central entregar a los estudiantes habilidades y capacidades que les permitan una mejor inserción en el mercado laboral (Mineduc, 2009). En este sentido, esta modalidad pretende ser una educación para el trabajo, adaptando los currículos a los requerimientos de los diferentes sectores económicos. Esto explica la existencia de distintas categorías dentro de la educación TP. Estas incluyen establecimientos educacionales TP con especialización Comercial, Industrial, Técnica, Agrícola y Marítima. Actualmente, la educación media TP representa un 33,2\% de la matrícula de educación secundaria en Chile (OECD, 2012) ${ }^{3}$.

La distinción entre educación $\mathrm{CH}$ y TP es relevante, pues en la práctica un alto porcentaje de los egresados de la educación secundaria no accede al sistema de educación superior. De hecho, Bassi y Urzúa (2010) muestran que mientras el 66\% de quienes cursan la modalidad CH continúa estudios terciarios, solo el $42 \%$ de los egresados de TP continúa sus estudios luego de obtener el certificado de educación media. Por otra parte, este estudio también documenta que menos de la mitad de quienes inician estudios terciarios, luego de haber cursado sus estudios secundarios en establecimientos de educación TP, los fi-

${ }^{2}$ Un esfuerzo reciente por rescatar la importancia de la educación TP fue la Comisión Asesora de Formación Técnico-Profesional que en 2008 convocó la entonces presidenta Michelle Bachelet. Dicha comisión destacó, entre otras cosas, la necesidad de integrar este tipo de educación en un marco de educación continua (Mineduc, 2009).

${ }^{3}$ De acuerdo a Farías y Sevilla (2012) en el año 2012 la participación de la educación TP alcanzaba un 43\%. Para efectos de la comparación con otros países utilizamos el dato entregado por la OECD que es comparable entre los distintos países. 
nalizan ${ }^{4}$. Por lo tanto, la educación secundaria es el último nivel al que muchos individuos acceden, constituyendo así la principal formación al momento de integrarse al mercado laboral.

La participación que tiene en Chile la educación media TP respecto del total de los estudiantes de secundaria contrasta con la realidad en los países desarrollados. A modo de ejemplo, en los países miembros de la Organización para la Cooperación y el Desarrollo Económico (OECD, por su sigla en inglés) la participación promedio de este sector alcanza el 44\% de la matrícula de educación secundaria. En Alemania, país en donde el sistema de educación dual genera una fuerte asociación entre la formación para el trabajo y el currículum educacional, la participación de este sector representa el 51,5\% (OECD, 2012). Esta característica ha permitido a Alemania sostener su desarrollo industrial, de la mano de aumentos en la productividad de su fuerza laboral. Con todo, y si bien es difícil poder concluir a partir de las diferencias en estas cifras que Chile sufre un déficit en el número de estudiantes matriculados en este sector, es posible contrastar la atención que recibe la educación TP en los países desarrollados con la situación que ha experimentado el sector durante las últimas décadas en Chile.

Quizás estas diferencias han motivado los recientes esfuerzos respecto de la necesidad de fortalecer la educación TP en el país. A modo de ejemplo, la Ley de Presupuestos del 2013 incluyó 29.297 millones de pesos para financiar la adquisición de equipamiento para los establecimientos TP, además de los 3.212 millones de pesos destinados al Programa Educación Técnico-Profesional, para el fortalecimiento y articulación de este tipo de educación. En línea con estos esfuerzos, recientemente se han planteado reformas a la estructura de la educación TP en cuanto a las especialidades que se imparten y a la pertinencia de estas mismas (CNED, 2010).

Es posible además anticipar que el interés por este nivel educacional aumentará en el tiempo debido a la necesidad de contar con una fuerza laboral más orientada a ocupaciones técnicas. Así, es muy posible que año a año veamos nuevas inversiones en capital físico destinadas a colegios de distintas especialidades técnicas. Estos avances, sin dejar de ser positivos, tienen que analizarse desde una perspectiva

${ }^{4}$ Los autores utilizan la información de la Encuesta de Trayectorias Educativas y Laborales del 2008 que incluye a 4.500 jóvenes de 25 a 30 años de áreas urbanas de Chile 
económica. En particular, dichas decisiones de inversión deben realizarse a la luz de la evidencia de la eficacia y eficiencia de estas mismas en el cumplimiento de objetivos claros y definidos transparentemente. Sin embargo, si de algo carece hoy la investigación respecto de la educación TP en Chile es de evidencia que de soporte a los planes de inversión en el sector.

Este trabajo aporta en la generación de evidencia para focalizar mejor los recursos destinados a la educación TP. Específicamente, presenta evidencia respecto del efecto causal de haber cursado alguna de las modalidades de educación TP sobre el número de meses de empleo formal y sobre los ingresos del trabajo. Ambas dimensiones del desempeño en el mercado laboral deben ser utilizadas al evaluar la focalización de gasto en educación media TP y sus distintas categorías. Adicionalmente, nuestros resultados documentan la heterogeneidad de los distintos tipos de educación TP en Chile.

El trabajo se organiza de la siguiente forma. La sección dos presenta una breve revisión de la literatura. La sección tres explica la relevancia de la educación TP en Chile. La sección cuatro describe nuestros datos. La sección cinco presenta nuestra estrategia empírica. La sección seis presenta nuestros resultados. Finalmente, la sección siete concluye.

\section{Revisión de la literatura}

La literatura que analiza el retorno de la educación, típicamente medido por los años de educación, sobre el desempeño en el mercado laboral es extensa ${ }^{5}$. Lo mismo los estudios que analizan el impacto de graduarse de la educación media ${ }^{6}$. Sin embargo, el efecto de distintos tipos de educación secundaria ha sido menos explorada en la literatura. En particular, la distinción entre la educación orientada al trabajo y la modalidad académica orientada a continuar estudios ha entregado resultados mixtos en términos de rentabilidad en el mercado laboral ${ }^{7}$.

La evidencia para los Estados Unidos del retorno de la educación técnica, por ejemplo, es ambigua. Gustman y Steinmeier (1982) encuen-

${ }^{5}$ Véanse Card (1999) y Meghir y Rivkin (2011) para una revisión de esta literatura y sus resultados.

${ }^{6}$ Véase, por ejemplo, Heckman y LaFontaine (2006).

${ }^{7}$ Esta modalidad recibe en Chile el nombre de Técnico-Profesional. En otros países es llamada Educación Vocacional. 
tran evidencia de retornos positivos de este tipo de educación tanto en el número de semanas trabajadas al año como en el salario mensual, aunque los resultados se obtienen solo para algunos subgrupos de la población, por ejemplo, mujeres blancas en programas de negocios. Por otra parte, estudios más recientes encuentran un efecto positivo de este tipo de educación en grupos más amplios de la población. Bishop y Mane (2004), por ejemplo, muestran que el retorno salarial de la educación técnica ha aumentado desde los años 70, hecho que se explicaría por el aumento en la demanda por habilidades para el trabajo. Además, dicho trabajo encuentra efectos positivos de la educación técnica incluso luego de controlar por estudios superiores. Por otro lado, Meer (2007) muestra la importancia de considerar distintos tipos de educación técnica. En efecto, este estudio separa la educación técnica en dos categorías: una orientada a los negocios y otra a oficios técnicos. Los resultados sugieren que algunos estudiantes obtendrían mayores ingresos al transitar de una educación orientada a oficios a una orientada a los negocios.

Un punto a destacar es que solo Meer (2007) corrige por el sesgo de selección que puede producir la endogeneidad de la decisión del tipo de establecimiento educacional al que el individuo asiste. Esta cuestión es importante debido a que, incluso luego de corregir por una variedad de variables, pueden existir factores no observables que determinen la elección del tipo de educación secundaria. Estos mismos factores pueden ser determinantes importantes de los resultados laborales posteriores $^{8}$.

Existe también evidencia de retornos salariales para países en vías de desarrollo. Neuman y Ziderman (1999, 2003) muestran evidencia de retornos positivos para Israel, particularmente para algunos grupos minoritarios. En tanto, Moenjak y Worswick (2003) encuentran efectos positivos para Tailandia. Sin embargo, otros estudios muestran resultados menos optimistas. Newhouse y Suryadarma (2011) encuentran retornos positivos para mujeres que fueron a colegios públicos, pero no para otros grupos. Finalmente, otros estudios encuentran efecto nulo de este tipo de educación (Malamud y Pop-Eleches, 2010).

En resumen, la evidencia internacional no permite alcanzar conclusiones precisas respecto del impacto de la educación secundaria TP.

${ }^{8}$ La importancia de corregir por sesgo de selección en los Estados Unidos ha sido documentada ampliamente. Véase por ejemplo Trost y Lee (1984). 
Evidencia para Chile. En el caso de Chile, la literatura que analiza la relación entre el tipo de educación secundaria y el mercado laboral es casi inexistente. Prácticamente la totalidad de la evidencia analiza el retorno a la educación secundaria sin prestar atención al tipo de establecimiento educacional, mientras que los pocos estudios que discuten el tema no controlan por la endogeneidad de la decisión. Esto es particularmente preocupante si consideramos que para muchos jóvenes la enseñanza media constituye el ciclo terminal en su proceso de acumulación de capital humano en el sistema de educación formal. Por lo tanto, sus salarios dependerán fundamentalmente de los conocimientos aprendidos y las habilidades que desarrollen en este nivel.

Una excepción es Bassi y Urzúa (2010). Este trabajo analiza las características de los egresados de educación media TP en Chile. Sus resultados sugieren que esta es menos efectiva en la preparación académica y laboral de los estudiantes ${ }^{9}$. Sin embargo, este estudio también sugiere que la educación media TP parece generar tipos de habilidades distintos a los adquiridos en los establecimientos de educación $\mathrm{CH}$. Este punto es discutido más adelante.

El informe de la comisión Mineduc (2009) es otra fuente reciente de información. En dicho informe se analiza el estado de los estudiantes de educación TP haciendo uso de las encuestas CASEN ${ }^{10}$. Este estudio muestra que no hay diferencias significativas entre los egresados de educación media $\mathrm{CH}$ y TP en términos de resultados laborales. Además sugiere que las ventajas en términos de inserción laboral para los egresados de TP son marginales. Ambas conclusiones son opuestas a los resultados que más adelante presentaremos. Conclusiones similares respecto al retorno que ambos tipos de educación secundaria obtienen han sido sugeridas en los trabajos de Sapelli (2009) y Arellano y Braun (1999). La diferencia de nuestros resultados respecto de la literatura se explica por que nuestra estrategia empírica controla por la posible endogeneidad de la decisión del tipo de establecimiento de educación secundario al que asistió el individuo.

${ }^{9}$ Estos autores analizan la Encuesta de Trayectorias Educativas y Laborales del 2008 que incluye información retrospectiva que permite caracterizar la historia educacional y laboral de la muestra.

${ }^{10}$ Las Encuestas de Caracterización Socioeconómica Nacional (CASEN) son encuestas de hogares, de carácter transversal y multipropósito. Estas encuestas son actualmente el principal instrumento de medición socioeconómica para el diseño y evaluación de la política social en Chile. 
En un estudio reciente, Bucarey y Urzúa (2013) analizan el retorno económico de la educación TP utilizando un modelo de Roy de dos sectores (Heckman y Honore, 1990). Específicamente, ese estudio presenta el retorno de este tipo de educación permitiendo la existencia de heterogeneidad en no-observables (Heckman, Urzúa y Vytlacil, 2006). En ese estudio se utiliza una estrategia de estimación alternativa al del presente trabajo, permitiendo que individuos observacionalmente equivalentes respondan de forma distinta al mismo tratamiento. Eso permite analizar desde otra perspectiva el impacto de la educación TP, complementando los resultados aquí analizados.

Finalmente, otro trabajo que intenta dar luces sobre el rol de la educación media TP es el de Iruarrizaga (2009) quien, utilizando datos de SIMCE en octavo básico y segundo medio, analiza las trayectorias y características de los estudiantes de educación media TP. Este trabajo encuentra que los estudiantes de TP provienen de familias más vulnerables y que el $70 \%$ de los alumnos de educación media asiste a un colegio en la comuna en que vive. Como veremos a continuación, esta última cifra es particularmente importante en el contexto de nuestra estrategia de identificación.

\section{La educación media Técnico-Profesional en Chile}

En Chile, al concluir la educación básica, los estudiantes enfrentan diferentes alternativas académicas respecto de dónde continuar su educación secundaria ${ }^{11}$. En efecto, deben escoger entre un colegio que imparta educación Técnico-Profesional (TP) o Científico-Humanista $(\mathrm{CH})^{12}$. Tal como fue discutido anteriormente, la opción $\mathrm{CH}$ corresponde a la modalidad en la cual se profundizan los contenidos de formación general, y que, a diferencia de la enseñanza TP, no tiene como foco principal la inserción laboral de sus alumnos.

Los colegios TP, por otra parte, se clasifican en distintas categorías. Estas pueden ser Comercial, Industrial, Técnico, Agrícola y Marí-

${ }^{11}$ En la práctica solo el $27 \%$ de los alumnos permanece en el mismo colegio entre octavo y tercero medio, por lo que de verdad existe movilidad entre octavo y primero medio (Iruarrizaga, 2009).

${ }^{12}$ Los colegios Científico-Humanista y los colegios Técnico-Profesional imparten enseñanza general en primero y segundo medio, y en estos últimos colegios las especialidades son impartidas en tercero y cuarto medio. 
timo. Además, un mismo colegio puede impartir una combinación de especialidades de los diferentes tipos de enseñanza antes mencionados, lo que es considerado en nuestro análisis empírico. La categoría de educación TP Comercial está asociada al sector económico de administración y comercio (una especialidad que ejemplifica esto es contabilidad). La enseñanza TP Industrial está asociada a sectores como electricidad y minería, con especialidades como metalurgia extractiva. La enseñanza TP Técnica tiene especialidades ligadas a hotelería y turismo o alimentación, en tanto, los colegios TP Agrícola están asociados a labores del sector agropecuario y maderero. Finalmente, los estudiantes que asisten a establecimientos educacionales TP Marítimo estudian especialidades como acuicultura y pesca. Como vemos los distintos tipos de enseñanza TP difieren significativamente en función del sector económico, pero están unidos por el foco en la inserción laboral.

La Figura $\mathrm{N}^{\circ} 1$ muestra el porcentaje de estudiantes matriculados en cada tipo de educación secundaria en el año $2000^{13}$. Observamos que la mayoría de los individuos siguieron la categoría $\mathrm{CH}$. El total de personas que estudió alguna especialidad TP (en cualquier categoría) corresponde a un 44,5\%. Cabe notar además que los individuos que cursaron alguna especialidad dentro de las categorías agrícola o marítima representan una proporción menor dentro del sistema, 2,4\% y $0,8 \%$, respectivamente. Esto se debe en parte a que este tipo de colegios se ubica solamente en algunas regiones y comunas, es decir, este tipo de educación está fuertemente segregada regionalmente, cuestión que confirmamos en el Cuadro $\mathrm{N}^{\circ} 1$. A modo de ejemplo, las cifras en este cuadro muestran que en el año 2000 la oferta de establecimientos TP Agrícolas y Marítimos es baja en comparación con el resto de las categorías.

Así, los estudiantes enfrentan un conjunto de alternativas que, en teoría, parece bastante amplio. Sin embargo, en la práctica la decisión de los alumnos al transitar de la educación básica a la media se encuentra acotada por las características de la oferta. En efecto, en muchas comunas de Chile solo se imparten algunos tipos de enseñanza o existe un número limitado de colegios que imparten alguno de los tipos de educación o especialidades. Esto limita las alternativas de los alumnos.

${ }^{13}$ La fuente de información para los datos en esta figura son del Ministerio de Educación. 
FIGURA N ${ }^{\circ}$ 1: PORCENTAJE DE PARTICIPACIÓN DE CADA TIPO DE EDUCACIÓN EN LA MATRÍCULA TOTAL DE EDUCACIÓN SECUNDARIA EN EL AÑO 2000

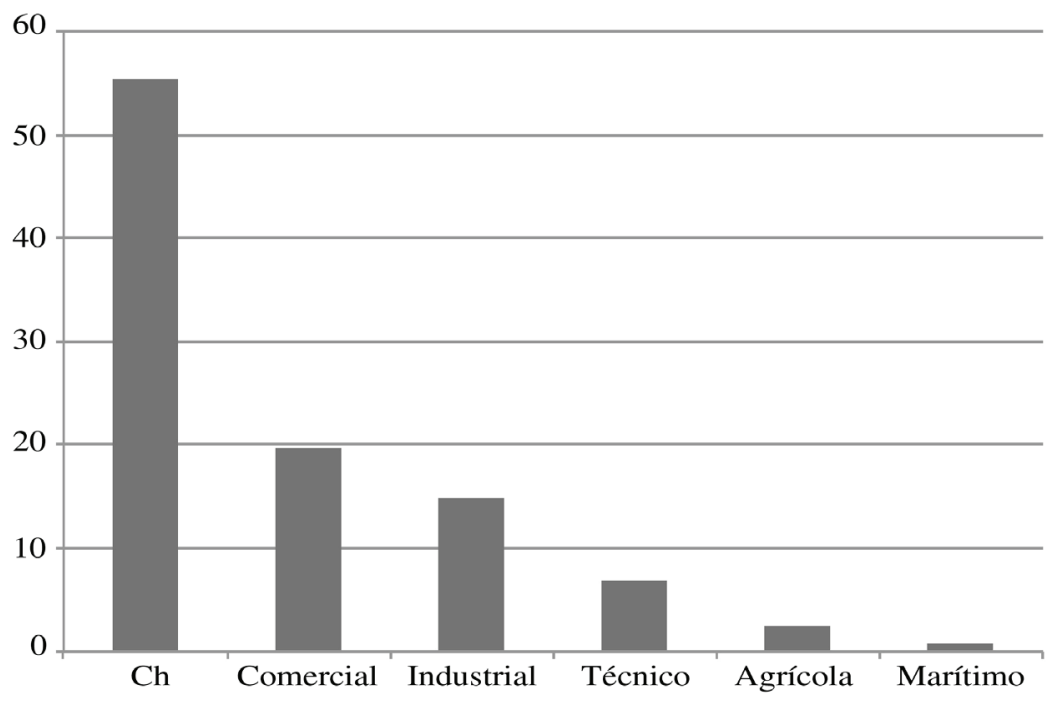

Nota: Los datos de Mineduc permiten identificar el número de alumnos en cada categoría pese a que asistan a colegios que combinen más de una categoría (híbridos). Fuente: Mineduc.

El Cuadro A en el apéndice presenta el número de jóvenes por tipo de educación TP para todas las comunas de Chile en el año 2000. Los resultados muestran que solo 142 de las 342 comunas ofrecen educación TP comercial, 147 industrial, 125 técnica, 103 agrícola y 28 marítima ${ }^{14}$. Nuestra estrategia empírica se beneficia de estas restricciones de oferta para estimar el impacto de la educación TP sobre empleo y salarios.

Tal como se mencionó anteriormente, otro elemento relevante al distinguir por tipo de educación secundaria son las diferencias en las habilidades y capacidades de los estudiantes. Estas pueden explicar algunas de las diferencias en el desempeño laboral de los individuos. El Cuadro $\mathrm{N}^{\circ} 2$ muestra las diferencias en los resultados de las prue-

${ }^{14}$ El número de comunas corresponde a la antigua división políticoadministrativa. El número de comunas al año 2013 es de 346. 
CUADRO N ${ }^{\circ}$ 1: PROMEDIO COMUNAL DEL NÚMERO DE COLEGIOS Y PROMEDIO COMUNAL DE LA PROPORCIÓN DE LA MATRÍCULA QUE CADA TIPO DE COLEGIO REPRESENTA EN LA MATRÍCULA TOTAL COMUNAL, POR REGIÓN

\begin{tabular}{lccccccc}
\hline & C-H & Comercial & Industrial & Técnico & Agrícola & Marítimo & Híbrido \\
\hline I & 6,83 & 2,17 & 1,17 & 1,00 & 0,33 & 0,17 & 1,83 \\
\%matrícula & 49,54 & 3,86 & 0,43 & 0,00 & 11,58 & 0,67 & 33,91 \\
II & 5,71 & 1,14 & 1,29 & 1,00 & 0,14 & 0,00 & 1,57 \\
\%matrícula & 26,29 & 2,53 & 1,54 & 1,31 & 14,29 & 0,00 & 54,05 \\
III & 2,88 & 1,38 & 1,38 & 0,25 & 0,13 & 0,38 & 2,13 \\
\%matrícula & 6,13 & 1,63 & 0,00 & 0,00 & 0,65 & 0,00 & 91,60 \\
IV & 4,77 & 0,62 & 0,54 & 0,46 & 0,46 & 0,15 & 1,00 \\
\%matrícula & 41,53 & 1,67 & 4,71 & 0,75 & 0,47 & 0,00 & 50,88 \\
V & 6,71 & 0,71 & 0,74 & 0,63 & 0,31 & 0,26 & 1,29 \\
\%matrícula & 50,50 & 1,06 & 3,19 & 2,12 & 4,98 & 0,44 & 37,72 \\
VI & 3,25 & 0,39 & 0,29 & 0,21 & 0,36 & 0,00 & 0,54 \\
\%matrícula & 63,04 & 1,20 & 0,87 & 0,70 & 2,08 & 0,00 & 32,12 \\
VII & 3,00 & 0,57 & 0,61 & 0,57 & 0,89 & 0,04 & 1,04 \\
\%matrícula & 32,48 & 2,51 & 4,06 & 0,31 & 8,54 & 0,00 & 52,10 \\
VIII & 2,84 & 0,50 & 0,60 & 0,30 & 0,38 & 0,08 & 0,68 \\
\%matrícula & 48,37 & 2,39 & 3,34 & 0,55 & 1,95 & 0,00 & 43,39 \\
IX & 2,42 & 0,71 & 0,58 & 0,45 & 0,45 & 0,03 & 0,68 \\
\%matrícula & 51,85 & 4,21 & 1,83 & 2,92 & 5,43 & 0,00 & 33,76 \\
X & 2,59 & 0,46 & 0,46 & 0,51 & 0,62 & 0,30 & 0,84 \\
\%matrícula & 32,19 & 2,86 & 3,41 & 0,91 & 9,20 & 2,59 & 48,84 \\
XI & 2,00 & 0,40 & 0,60 & 0,00 & 0,20 & 0,40 & 0,80 \\
\%matrícula & 44,82 & 0,00 & 0,00 & 0,00 & 1,17 & 0,00 & 54,01 \\
XII & 5,00 & 1,25 & 1,25 & 0,75 & 0,00 & 0,00 & 0,75 \\
\%matrícula & 54,60 & 3,38 & 4,45 & 1,70 & 0,00 & 0,00 & 35,87 \\
Región Metr. & 10,90 & 2,83 & 2,31 & 1,48 & 0,21 & 0,00 & 2,42 \\
\%matrícula & 39,99 & 9,72 & 4,89 & 1,93 & 1,89 & 0,00 & 41,58 \\
\hline & & & & & & &
\end{tabular}

Nota: Distribución regional en 2000.

Fuente: Cálculos de los autores en base a información de Mineduc.

bas SIMCE para el año 2001 (segundos medios) entre los individuos provenientes de los diferentes tipos de educación. Los estudiantes de colegios TP logran menores puntajes en SIMCE, tanto en lenguaje como en matemáticas. Estas diferencias además se traducen en puntajes más bajos en las pruebas de selección universitaria, lo que se ha argumentado explica el menor acceso de los estudiantes de establecimientos TP al sistema de educación superior. 
CUADRO N 2: $\quad$ PUNTAJE SIMCE PROMEDIO 2001 Y AÑOS PROMEDIO DE ESCOLARIDAD DE LOS PADRES POR TIPO DE EDUCACIÓN

\begin{tabular}{lccrc}
\hline Tipo educación & $\begin{array}{c}\text { Simce 2001 } \\
\text { Lenguaje }\end{array}$ & $\begin{array}{c}\text { Simce 2001 } \\
\text { Matemáticas }\end{array}$ & $\begin{array}{c}\text { Escolaridad } \\
\text { madre }\end{array}$ & $\begin{array}{c}\text { Escolaridad } \\
\text { padre }\end{array}$ \\
\hline C-H & 263,1 & 259,9 & 11,6 & 12,0 \\
Comercial & 251,5 & 242,0 & 9,3 & 9,4 \\
Industrial & 239,8 & 241,3 & 9,3 & 9,3 \\
Técnico & 233,7 & 218,0 & 8,8 & 8,6 \\
Agrícola & 231,6 & 226,8 & 8,0 & 7,8 \\
Marítimo & 243,6 & 240,2 & 10,0 & 9,5 \\
Híbrido & 235,7 & 228,7 & 8,9 & 8,9 \\
Todos & 249,3 & 244,0 & 10,1 & 10,3 \\
No de observaciones & 152.607 & 152.607 & 121.733 & 113.290 \\
\hline
\end{tabular}

Fuente: Cálculo de los autores en base a datos Simce 2001.

Como vemos los alumnos de colegios TP parecen tener peor rendimiento académico respecto a los alumnos de $\mathrm{CH}$. Sin embargo, en la medida que el mercado valore otro tipo de habilidades y capacidades, es posible que esto no necesariamente implique un peor desempeño laboral. De hecho, Bassi y Urzúa (2010) muestran que los individuos que escogieron diferentes tipos de educación media difieren en sus capacidades y habilidades. Específicamente, estos autores presentan evidencia de que los egresados de educación TP tienen menos habilidades cognitivas que los egresados de $\mathrm{CH}$ pero poseen mayores habilidades socioemocionales (autosuficiencia, habilidades metacognitivas o de planificación de tareas y habilidades sociales). Como discutiremos a continuación, estos resultados pueden explicar la mayor empleabilidad de algunos tipos de educación TP.

\section{Datos}

Nuestros datos reúnen información a nivel individual para la cohorte de personas que en el año 2001 rindió la prueba SIMCE mientras cursaban segundo año medio. Estos mismos individuos son observados luego en el mercado laboral entre enero de 2008 y mayo de 2011, período para el cual contamos con información administrativa de salarios. Dicha información proviene del sistema del Seguro de Cesantía y por 
tanto, a diferencia de los estudios previos no son ingresos autodeclarados. Además, sabemos si estos individuos cursaron o no estudios terciarios entre 2007 y 2010.

Nuestras fuentes de información nos permiten disponer de datos para 152.607 personas con edades entre los 24 y 27 años. Debido a que analizaremos los resultados laborales en 2010, excluiremos a quienes ese año cursaban estudios terciarios. Esto debido a que quienes lo hacían no estaban completamente integrados al mercado laboral. El Cuadro $\mathrm{N}^{\circ} 3$ muestra las características de las 37.167 personas que son excluidas de la muestra original de 152.607. Vemos que en general son egresados de la modalidad $\mathrm{CH}$ y que al compararlos con el grupo que no cursaba estudios terciarios en 2010 (comparación de columna 1 y 2), se trata de individuos con mayor puntaje SIMCE y con padres con

\section{CUADRO N$^{\circ}$ 3: ESTADÍSTICA DESCRIPTIVA DE INDIVIDUOS MATRICULADOS EN} EDUCACIÓN TERCIARIA EN 2010 Y DIFERENCIAS CON OTROS GRUPOS

\begin{tabular}{lrrr}
\hline & $(1)$ & $(2)$ & $(3)$ \\
\hline Porcentaje TP & 26,07 & 41,74 & $-15,67^{* *}$ \\
Sexo & 43,90 & 49,31 & \\
& 0,52 & 0,52 & $0,01^{* *}$ \\
Simce Lenguaje & 0,50 & 0,50 & \\
& 270,30 & 242,47 & $27,83^{* * *}$ \\
Simce Matemáticas & 47,41 & 47,26 & \\
& 267,56 & 236,43 & $31,13^{* * *}$ \\
Años de escolaridad madre & 53,93 & 46,84 & \\
& 11,61 & 9,63 & $198^{* * *}$ \\
Año escolaridad padre & 4,85 & 4,76 & \\
& 11,90 & 9,80 & $2,10^{* * *}$ \\
No de meses de empleo en 2010 & 5,13 & 4,85 & \\
& 4,28 & 6,77 & $-2,49 * * *$ \\
Salario promedio en 2010 & 4,87 & 5,01 & \\
& 114.922 & 194.030 & $-79.108^{* * *}$ \\
$\mathrm{~N}^{\circ}$ de observaciones & $186.455,23$ & 218.837 & \\
\hline
\end{tabular}

(1): Individuos matriculados en educación terciaria en 2010.

(2): Individuos no matriculados en educación terciaria en 2010.

(3): Diferencia de medias entre (1) y (2).

Nota: Sexo toma valor 1 para hombres y 0 para mujeres. $* \mathrm{p}<0,1, * * \mathrm{p}<0,05$ y $* * * \mathrm{p}<0,01$. 
mayor escolaridad. Nuestra estrategia empírica considera estas diferencias. Específicamente nuestros modelos de regresión incluyen controles por ambas variables. Esto debe reducir los riesgos de potenciales sesgos producto de la exclusión del grupo de individuos con estudios terciarios.

En nuestros datos identificamos el tipo de enseñanza media impartida en el colegio al que asistían los individuos en 2001 y la comuna de dicho establecimiento. Los tipos de enseñanza que identificamos son TP categorizada en Comercial, Industrial, Técnico, Agrícola y Marítimo, además de la enseñanza Científico-Humanista. A su vez, dado que identificamos el colegio al que cada uno asistía pero no la especialidad que efectivamente cursó, generamos una categoría adicional que llamamos Híbrido, que corresponde a los colegios que imparten más de uno de los tipos de enseñanza. Los alumnos en establecimientos de esa categoría pueden pertenecer a cualquiera de las especialidades de la educación TP o a la enseñanza $\mathrm{CH}$.

La Figura $\mathrm{N}^{\circ} 2$ muestra el porcentaje de estudiantes matriculados en cada tipo de establecimiento educacional secundario en nuestra muestra. Los resultados siguen el mismo patrón observado en la Figura $\mathrm{N}^{\circ} 1$, aun cuando en este caso se incluye la categoría de establecimientos Híbridos. Así, la mayoría de individuos estudiaron en colegios $\mathrm{CH}$. El total de personas que provienen de un liceo TP (cualquier categoría) corresponde al $25,2 \%{ }^{15}$.

Finalmente, para implementar la estrategia empírica que detallamos en la siguiente sección utilizamos variables de oferta de los distintos tipos de enseñanza a nivel comunal en el año 2000, datos que obtenemos del Ministerio de Educación (Mineduc). En particular, utilizamos el número de colegios por comuna que imparte cada tipo de enseñanza y la proporción de la matrícula total comunal que representa cada tipo de enseñanza.

Resultados preliminares. El Cuadro $\mathrm{N}^{\circ} 2$ confirma las diferencias en SIMCE 2001 y educación de los padres entre los individuos provenientes de los diferentes tipos de educación en nuestra muestra. Los individuos provenientes de colegios TP alcanzan menores puntajes

${ }^{15}$ Esta cifra difiere de las cifras oficiales de participación en educación TP debido a que parte de los programas que categorizamos como tipo Híbrido corresponden a educación TP. 
FIGURA N ${ }^{\circ}$ 2: $\quad$ TIPO DE EDUCACIÓN SECUNDARIA QUE ESTUDIARON LOS INDIVIDUOS DE LA MUESTRA (PORCENTAJES)

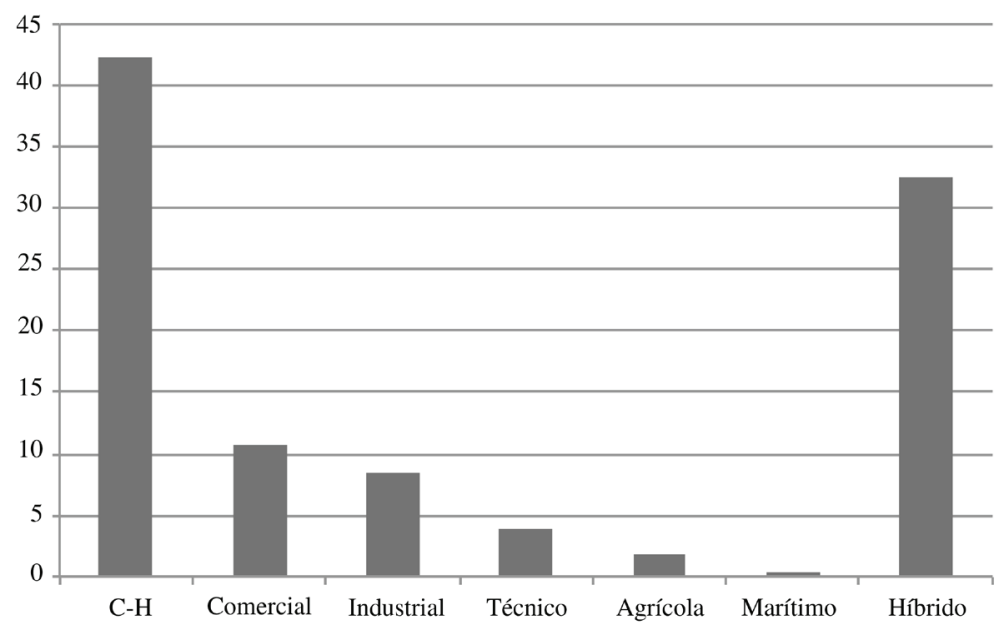

Fuente: Elaboración propia en base a datos del Seguro de Cesantía.

en SIMCE y sus padres poseen menores niveles de escolaridad en promedio. Además, como vemos en la Figura $N^{\circ} 3$ las tasas de matrícula en educación terciara también difieren entre los distintos tipos de educación secundaria. Con todo, los alumnos de colegios TP tienen padres menos educados, están rezagados en el desempeño académico y tienen menor acceso al sistema de educación superior que los alumnos de establecimientos $\mathrm{CH}$.

La Figura $\mathrm{N}^{\circ} 4$ presenta las diferencias salariales en el año 2010 por el tipo de establecimiento educacional al que asistieron los individuos en el año 2001. Las cifras se presentan para dos definiciones alternativas de ingresos del trabajo: el promedio mensual de 12 meses de salario durante el año 2010, que corresponde a las barras más oscuras en la figura, y el promedio de la remuneración en los meses en que los individuos tuvieron trabajo, las barras grises. Así, si consideramos las remuneraciones promedio anuales, observamos que los individuos que cursaron la categoría Industrial tienen un salario 
FIGURA N ${ }^{\circ}$ 3: $\quad$ TASAS DE MATRÍCULA PROMEDIO POR TIPO DE EDUCACIÓN

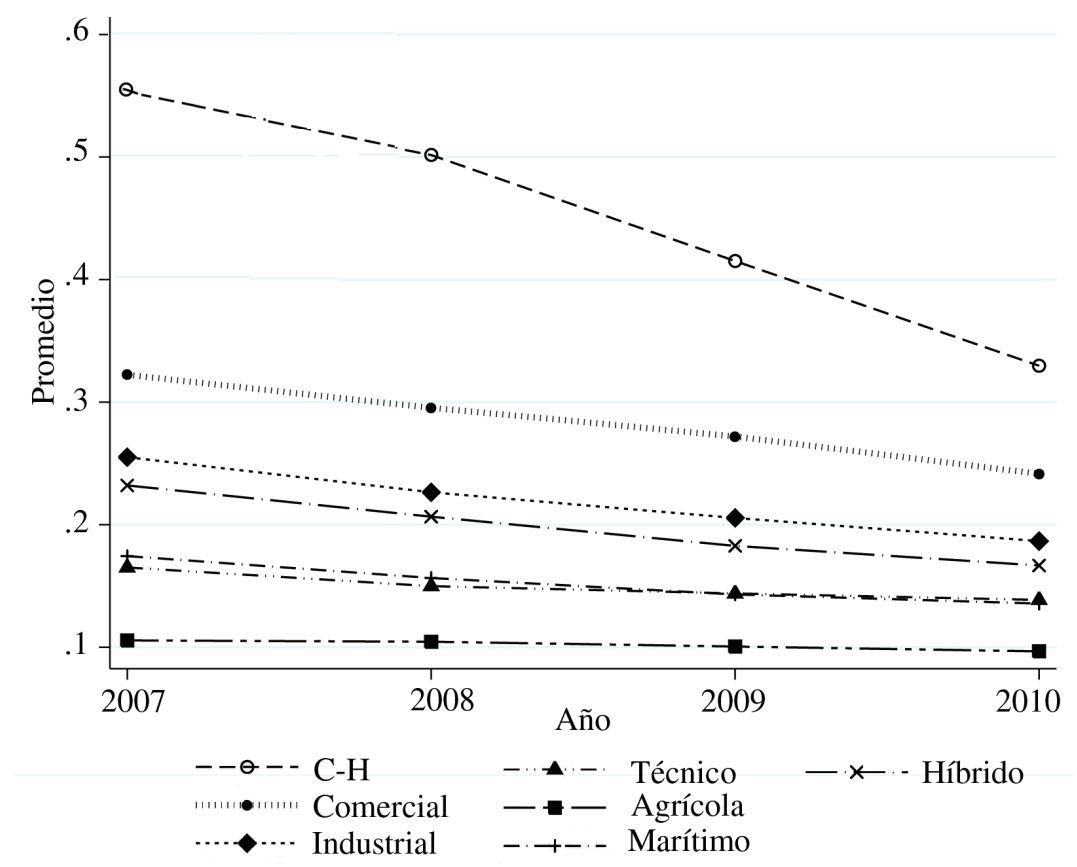

Nota: La matrícula que consideramos es para cualquier institución de educación terciaria (universidad y CFT).

Fuente: Cálculo de los autores en base a datos del Seguro de Cesantía.

promedio $32 \%$ mayor que el de quienes cursaron estudios en un colegio $\mathrm{CH}$. En tanto, al considerar el promedio de remuneraciones de los meses durante los cuales los individuos contaron con empleo, ambos tipos de educación obtienen un salario similar. Es claro que esta diferencia se debe a las tasas de empleo asalariado formal que muestran los diferentes grupos y que podemos apreciar en la Figura $\mathrm{N}^{\circ}$ 5. Esta figura muestra el número de meses promedio sin empleo formal para el período enero 2010 y mayo 2011 para nuestra muestra. Vemos que los egresados de liceos $\mathrm{CH}$ son quienes en promedio presentan un mayor desempleo en el sector formal ( 7,8 meses) y quienes presentan un mayor empleo en el sector formal son los egresados de liceos TP 
FIGURA N ${ }^{\circ}$ 4: REMUNERACIONES AÑO 2010 POR TIPO DE EDUCACIÓN ENTRE QUIENES NO ESTÁN MATRICULADOS EN 2010 EN EDUCACIÓN TERCIARIA (EN PESOS CHILENOS)

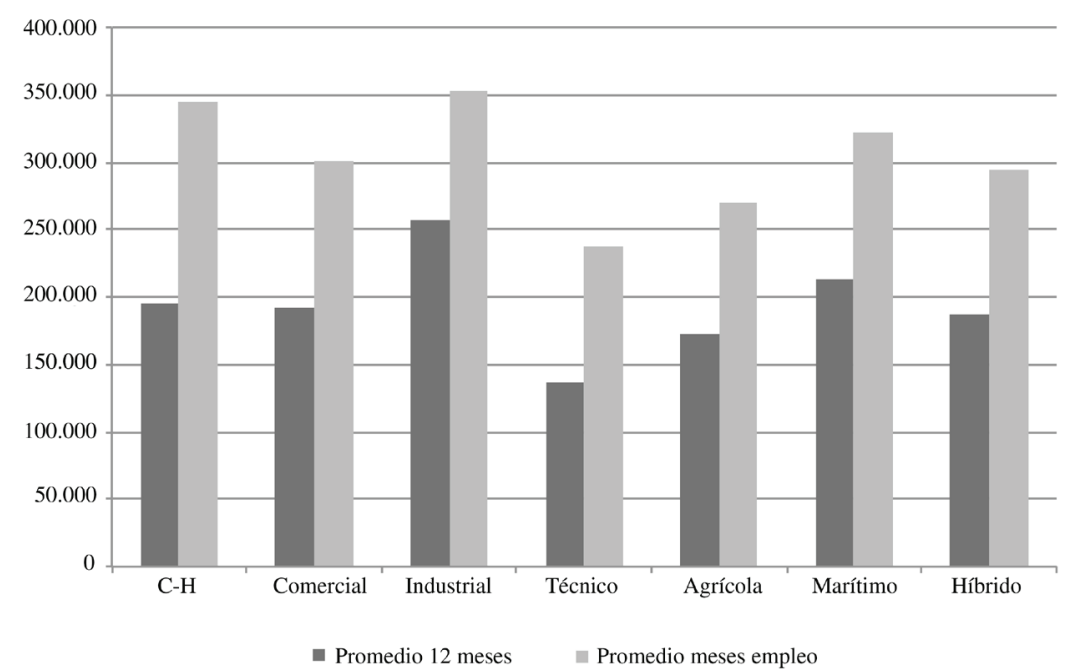

Nota: La matrícula que consideramos es para cualquier institución de educación terciaria (universidad y CFT). Promedio meses empleo corresponde al salario total anual dividido por el número de meses trabajados.

Fuente: Cálculos de los autores en base a datos del Seguro de Cesantía.

FIGURA N ${ }^{\circ}$ 5: DESEMPLEO PROMEDIO ENTRE ENERO 2010 Y MAYO 2011 ENTRE QUIENES NO ESTÁN MATRICULADOS EN 2010 EN EDUCACIÓN TERCIARIA

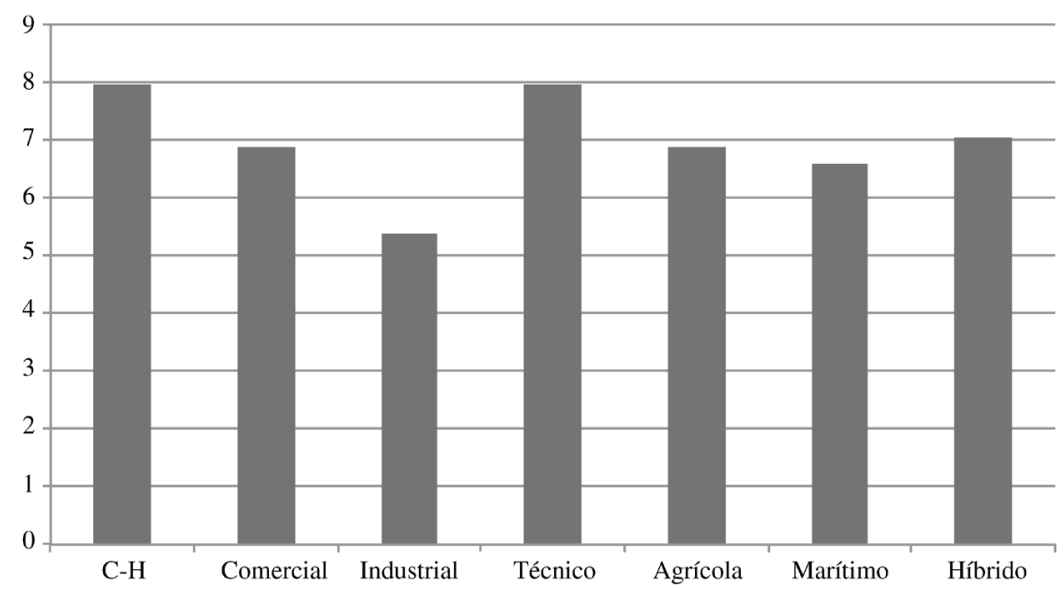

Nota: La matrícula que consideramos es para cualquier institución de educación terciaria (universidad y CFT).

Fuente: Cálculos de los autores en base a datos del Seguro de Cesantía. 24-3-2013 
industriales, alcanzando solo 5,4 meses promedio de desempleo en el sector formal en el lapso de 17 meses analizado. Resulta evidente entonces que el desempleo dentro de la cohorte de individuos que analizamos es un factor importante. La Figura $\mathrm{N}^{\circ} 6$ profundiza este punto mostrando el porcentaje de individuos que exhibe un desempleo continuado de 17 meses entre enero de 2010 y mayo de 2011 dentro de los egresados de cada tipo de educación secundaria. Por ejemplo, dentro de los egresados de liceos $\mathrm{CH}$ el $21 \%$ no estudia ni trabaja formalmente en el período enero 2010 a mayo 2011. La contraparte de este análisis es que los egresados de $\mathrm{CH}$ son quienes en menor porcentaje tienen trabajo durante los 17 meses continuados que analizamos, de hecho solo el 25\% 12 de ellos tiene trabajo formal durante todo el período.

La evolución de los salarios también difiere por tipo de educación. Las Figuras Nos. 7 y 8 muestran las trayectorias de los salarios

FIGURA N ${ }^{\circ}$ 6: PORCENTAJE DE DESEMPLEADOS DURANTE TODO EL PERIODO ENERO 2010 A MAYO 2011 ENTRE QUIENES NO ESTÁN MATRICULADOS EN 2010 EN EDUCACIÓN TERCIARIA

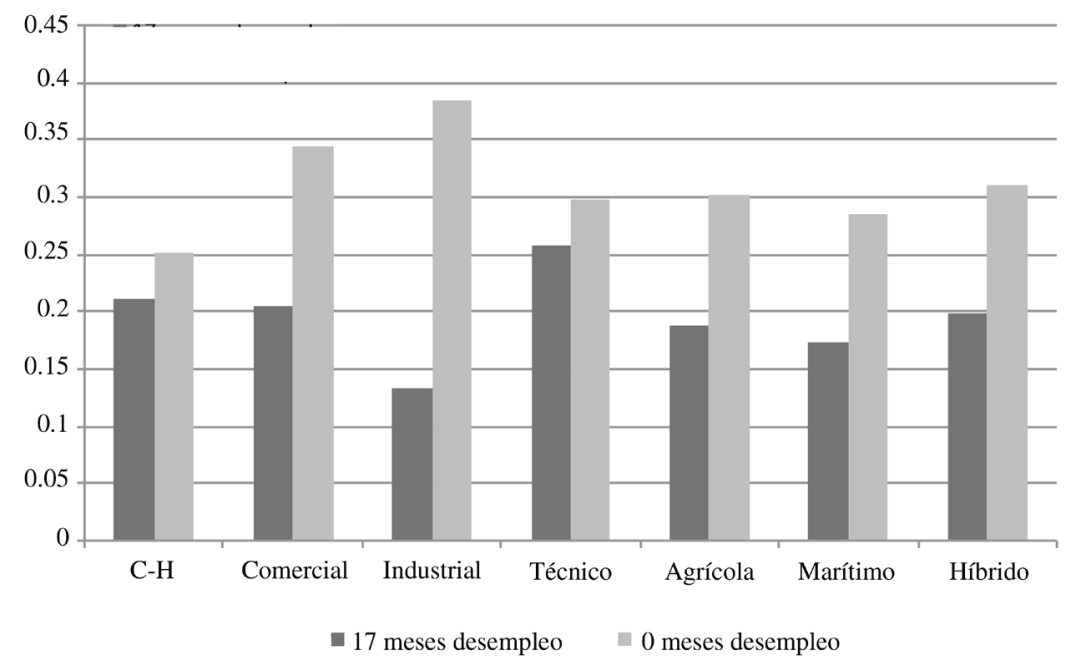

Nota: La matrícula que consideramos es para cualquier institución de educación terciaria (universidad y CFT).

Fuente: Cálculos de los autores en base a datos del Seguro Cesantía. 
FIGURA N ${ }^{\circ}$ 7: $\quad$ REMUNERACIONES PROMEDIO POR MES Y TIPO DE EDUCACIÓN SECUNDARIA

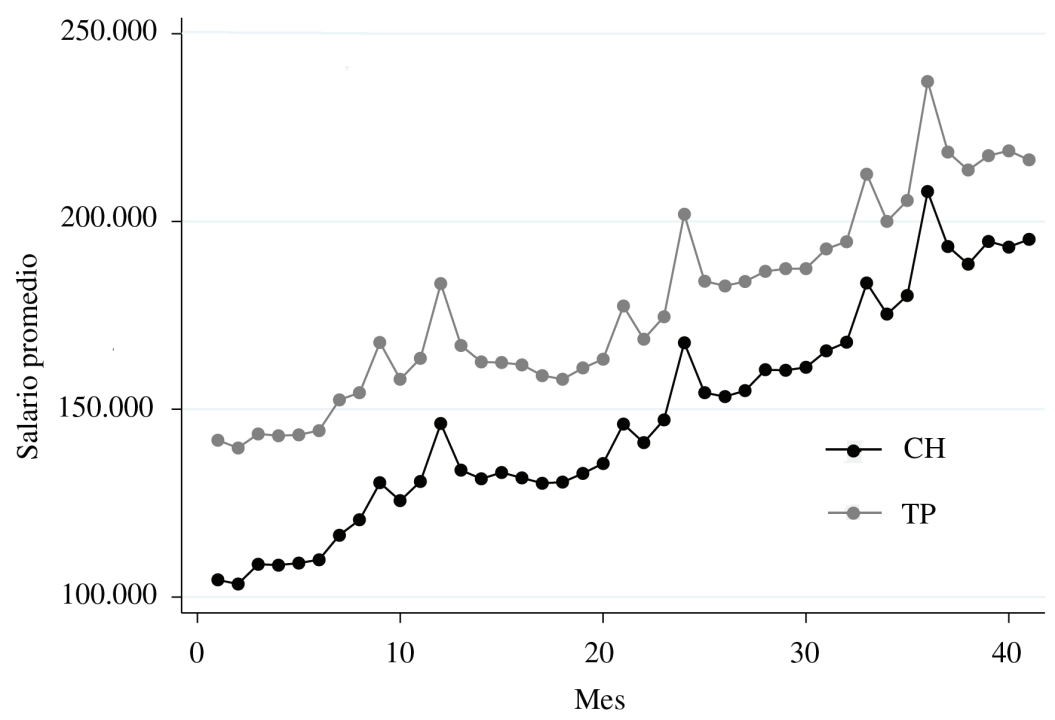

Fuente: Cálculos de los autores en base a datos del Seguro C'esantia.

FIGURA N ${ }^{\circ}$ 8: $\quad$ REMUNERACIONES PROMEDIO POR MES Y TIPO DE EDUCACIÓN SECUNDARIA (mes 1 corresponde a enero 2008)

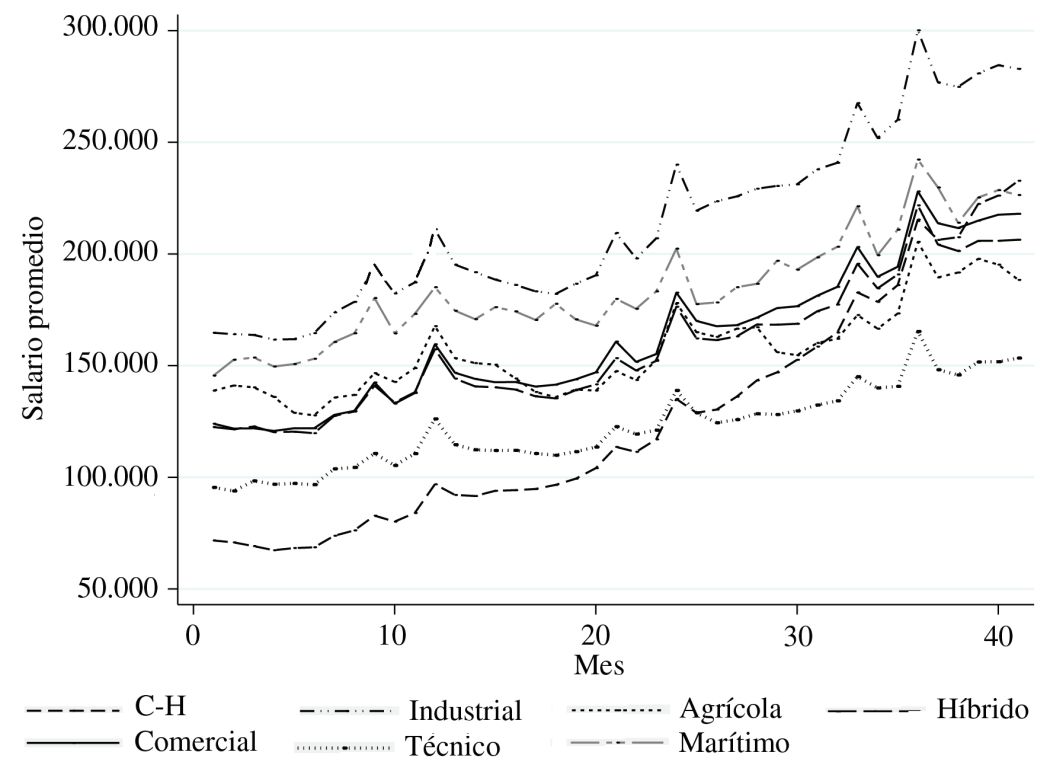

Fuente: Cálculos de los autores en base a datos del Seguro Cesantía. 
promedio para los diferentes grupos. Vemos que los salarios promedio entre $\mathrm{CH}$ y TP tienden a igualarse, pero al mirar el desglose de tipos de educación TP el panorama es mixto. Un caso interesante es el de quienes estudiaron en un colegio TP Industrial. Este grupo presenta remuneraciones mensuales muy por sobre el resto, hecho que corroboramos con el análisis de la siguiente sección.

Una particularidad de nuestros datos, y que podría afectar la representatividad de nuestros resultados, es que provienen solamente de trabajadores asalariados y con contrato formal. De este modo, podríamos estar sobrerrepresentando grupos con menores tasas de informalidad, afectando consecuentemente la comparación entre quienes asistieron a establecimientos $\mathrm{CH}$ versus TP. Sin embargo, utilizando los datos de la Encuesta de Trayectorias Educativas y Laborales del 2008 mostramos que los egresados de liceos $\mathrm{CH}$ son bastante similares a los egresados de TP en cuanto al porcentaje de individuos que trabajan de manera informal ${ }^{16}$. En efecto, la Figura $\mathrm{N}^{\circ} 9$ muestra que el porcentaje de personas que tiene un trabajo informal es similar entre TP y $\mathrm{CH}$. De

FIGURA No 9: $\quad$ TIPO DE CONTRATO

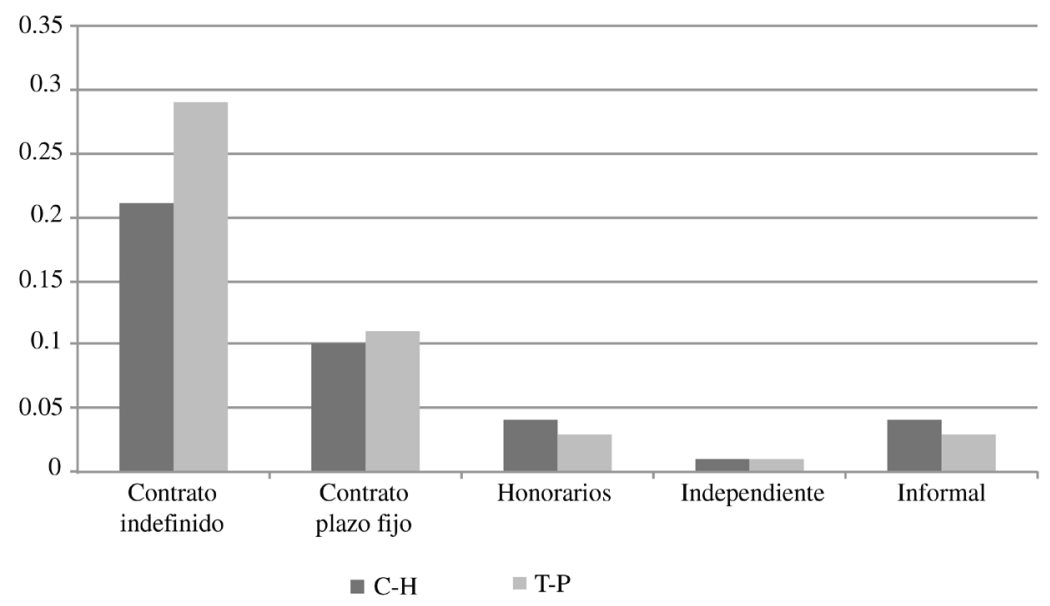

Fuente: Cálculo de los autores en base a ETEL 2008

${ }^{16}$ La encuesta incluye a 4.500 jóvenes de 25 a 30 años de áreas urbanas de Chile y fue desarrollada por el Banco Interamericano de Desarrollo. Véase Bassi y Urzúa (2010) para mayores detalles. 
este modo, consideramos que los posibles problemas de representatividad pueden no ser severos.

En la siguiente sección presentamos la estrategia empírica con la que luego obtenemos los resultados formales del efecto de haber cursado educación TP sobre empleabilidad y salarios.

\section{Estrategia empírica}

En esta sección consideramos el siguiente modelo de regresión para estimar el impacto de asistir a un establecimiento de educación secundaria TP sobre el desempeño en el mercado laboral:

$$
Y_{i}=\alpha_{1}+\rho T P_{i}+X_{i} \beta+\varepsilon_{i}
$$

en donde $Y_{i}$ corresponde a un determinado resultado en el mercado laboral (número de meses de empleo formal, salario promedio mensual anual ${ }^{17}$ o salario promedio en los meses en que el individuo contó con empleo asalariado formal ${ }^{18}$ ); $T P_{i}$ corresponde a una variable binaria que indica si el individuo cursó segundo año medio en un liceo $T P ; X_{i}$ es un vector con características del individuo (habilidades medidas antes de la entrada al mercado laboral, la escolaridad de la madre, la dependencia del establecimiento en que estudió, dummies por región de residencia y variables que controlan por matrícula en educación terciaria entre 2007 y 2010$)$, y $\varepsilon_{i}$ es el término de error. En este contexto, $\rho$ denota al parámetro de interés.

Sin embargo, la decisión de asistir a un establecimiento TP no es exógena. Esta puede depender de factores no observables que podrían generar un problema de endogeneidad, lo que implicaría la existencia de sesgos al estimar la ecuación (1) vía Mínimos Cuadrados Ordinarios (MCO). Para hacernos cargo de la endogeneidad de esta variable utilizamos como instrumento la oferta de colegios TP a nivel comunal en el

${ }^{17}$ Corresponde a la suma del salario de los 12 meses del año dividido por 12 independientemente de si el individuo trabajó o no los 12 meses, de modo que a quienes no trabajaron se les asignó un salario promedio de 0 .

${ }^{18}$ Que corresponde al promedio del salario considerando solo los meses trabajados del año, de modo que quien no trabajó durante el año no participa en el cálculo. 
año 2000. Esta es una estrategia común en la literatura ${ }^{19}$. Su lógica es simple. La oferta de establecimientos afecta la probabilidad de asistir a ellos, pero no necesariamente afecta el salario 10 años después de haber egresado (luego de controlar por el vector X).

Un segundo elemento que no considera la ecuación (1) es la existencia de distintos tipos de educación TP. Esto no permite capturar la posible heterogeneidad en los retornos en función del tipo de establecimiento educacional TP. Para efectos de capturar este elemento, definimos $\mathrm{S}$ como el número de tipos de establecimientos y extendemos el modelo de regresión de la siguiente forma:

$Y_{i}=\alpha_{1}+\sum_{j=1}^{S} \rho_{j} T P_{j i}+X_{i} \beta+\varepsilon_{i}$

en donde $T P_{j i}$ es una variable binaria que indica si el individuo $i$ cursó segundo año medio en un liceo del tipo $j(\operatorname{con} j=\{1, \ldots, S\})$. Sin embargo, el problema de selección ahora es aún más complejo, pues ahora se tienen $S$ potenciales variables endógenas. Para controlar por esto, utilizamos como instrumentos la oferta de colegios de cada tipo de educación a nivel comunal en el año 2000. La oferta relevante para el individuo es circunscrita a la comuna a la que asistía en segundo medio el año 2001. Dado lo anterior, estimaremos el efecto de asistir a educación TP de cada tipo aprovechando la variación en la oferta educativa a nivel comunal. El modelo empírico es descrito por la ecuación (2) y por la estimación de las regresiones:

$T P_{j i}=\alpha_{2}+\sum_{h=1}^{S} \delta_{l j} N_{j i}+\sum_{h=1}^{S} \delta_{2 j} \operatorname{Prop}_{j i}+X_{i} \gamma+v$

donde $j=1, . ., S . N_{j i}$ y $\operatorname{Prop}_{j i}$ corresponden a los instrumentos. $N_{j i}$ representa al número de colegios que impartían la categoría $j$ en el 2010 en la comuna en que el individuo $i$ cursó segundo medio, mientras que Prop $_{j i}$ representa la proporción de la matrícula total que comprendía el tipo de educación $j$ en la misma comuna y año antes mencionados. Nuestro

${ }^{19}$ Véase Card (2001) para mayor detalle sobre el uso y justificación de esta estrategia. 
supuesto para la identificación del efecto de $T P_{j}$ sobre el resultado $Y$ es que estas variables (instrumentos) proveen una variación exógena que afecta el conjunto de opciones que tiene el estudiante que ingresa a primero medio. En este sentido, asumimos que la oferta comunal de establecimientos influye en la decisión del tipo de colegio en el que el alumno cursará la educación secundaria. Este último es un supuesto razonable, pues se ha documentado, por ejemplo, que en 2007 el $70 \%$ de los alumnos que cursaba tercero medio asistía a un colegio dentro de su comuna de residencia (De Iruarrizaga, 2009), por lo que el supuesto de que la oferta relevante está en la comuna del colegio al que asistió el estudiante en segundo medio no parece muy fuerte.

Un análisis geográfico del número de colegios muestra que hay diferencias importantes en la oferta entre las diferentes comunas. La Figura $\mathrm{N}^{\circ} 10$ muestra los resultados para la zona central de Chile. Las comunas blancas indican que no hay colegios que impartan educación TP agrícolas en el año 2000, en tanto que en las comunas negras hay entre dos y tres de estos colegios ${ }^{20}$. Así, las diferencias entre las comunas en la oferta de enseñanza TP son evidentes. Hay comunas en que los estudiantes no cuentan con la opción de escoger un determinado tipo de educación TP. Este hecho motiva nuestra estrategia de identificación del efecto de este tipo de educación en salarios y empleo. Esta variación en la oferta a nivel comunal se repite a lo largo del país y en los diferentes tipos de educación existiendo un patrón de especialización geográfica. En efecto, los colegios que imparten especialidades agrícolas se encuentran en sectores rurales, en tanto, los que imparten especialidades marítimas se concentran en zonas costeras ${ }^{21}$. El Cuadro $\mathrm{N}^{\circ} 1$, analizado anteriormente, confirma la existencia de heterogeneidad entre regiones en el número promedio de colegios que existen de cada tipo de enseñanza y la proporción promedio de la matrícula que cada tipo de enseñanza

${ }^{20}$ El conjunto completo de resultados gráficos por región puede ser solicitado a los autores.

${ }^{21}$ Una crítica válida a nuestra estrategia de identificación es que la ubicación geográfica de los establecimientos educacionales no es exógena respecto de las condiciones del mercado laboral. Sin embargo, dado que los resultados laborales que analizamos se observan casi una década después de haber egresado de la educación secundaria, consideramos que esta crítica puede no afectar a nuestros resultados de forma significativa. De todos modos, el estudio de la ubicación geográfica de los establecimientos educacionales es un tema que debe ser considerado por la literatura. 
FIGURA N ${ }^{\circ} 10$ : NÚMERO DE COLEGIOS AGRÍCOLAS ZONA CENTRAL

(V a VIII región en 2000)

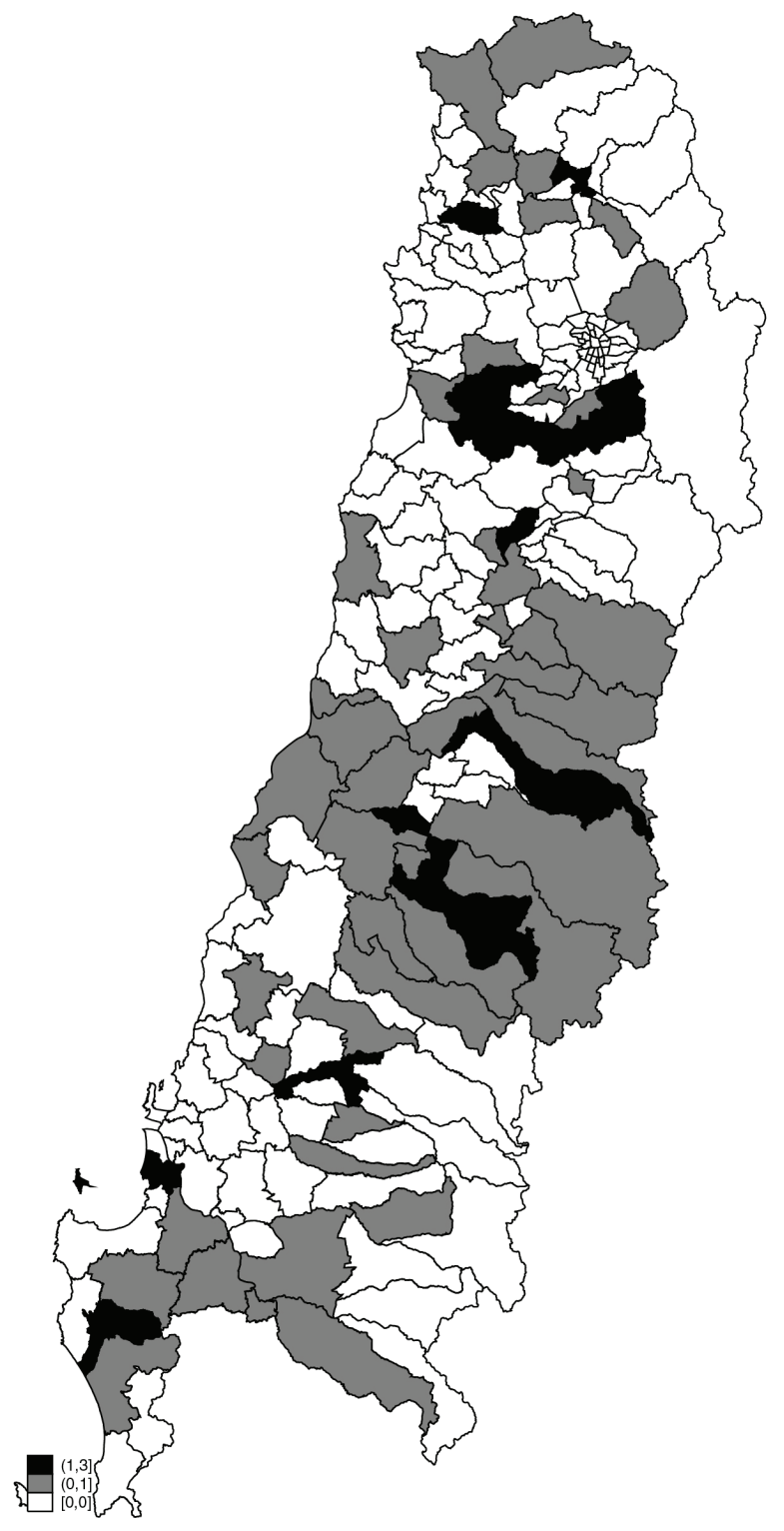

Fuente: Cálculos propios en base a información de Mineduc. 
representa dentro de las comunas. El Cuadro $\mathrm{N}^{\circ} 4$ documenta que hay un número importante de comunas que no cuentan con establecimientos que impartan determinados tipos de educación. Por ejemplo, 157 comunas no poseen colegios TP industriales.

CUADRO N 4: NÚMERO DE COLEGIOS POR COMUNA Y TIPO DE EDUCACIÓN

\begin{tabular}{lrrr}
\hline Tipo/Número de colegios & 0 & 1 & 2 \\
\hline C-H & 7 & 138 & 54 \\
Comercial & 162 & 77 & 30 \\
Industrial & 157 & 89 & 28 \\
Técnico & 179 & 84 & 25 \\
Agrícola & 201 & 84 & 17 \\
Marítimo & 276 & 24 & 2 \\
Híbrido & 96 & 140 & 36 \\
\hline
\end{tabular}

Notas: El número total de comunas consideradas es 341.

Fuente: Cálculos de los autores en base a información Mineduc.

\section{Resultados}

Comenzamos analizando el efecto de haber cursado alguna categoría de educación TP en el número de meses de empleo formal en el año 2010.

El Cuadro $\mathrm{N}^{\circ} 5$ presenta los resultados sin separar por categoría de educación TP. Los resultados obtenidos a través de MCO sugieren un efecto de 0,8 meses adicionales asociados a haber estudiado en algún tipo de educación TP. Sin embargo, tal como se mencionó antes, la potencial endogeneidad de la decisión del tipo de educación media al que asistió el individuo puede sesgar esta estimación. Al corregir por sesgo de selección mediante el uso de variables instrumentales vemos que el efecto disminuye levemente, indicando un efecto de 0,7 meses adicionales de emppleo (columna 3 ). Cabe notar que en nuestra estimación del efecto, intencionadamente no controlamos por la matrícula en educación terciaria entre 2007 y 2009 debido a que luego de controlar por la habilidad capturada por la prueba SIMCE y al corregir la endogeneidad usando variables instrumentales, el efecto de asistir a un colegio TP captura un "paquete completo" que incluye el efecto asociado a las menores posibilidades de asistir a educación terciaria. 
Con el propósito de mostrar la sensibilidad de nuestros resultados respecto del punto anterior, estudiamos el efecto sobre empleo para el subgrupo de personas que no continuó estudios terciarios en el período 2007-2010. Nuevamente presentamos los resultados de MCO y VI en el Cuadro $\mathrm{N}^{\circ}$ 5. El primer estimador confirma un efecto de 0,6 meses de empleo adicional por año y la estimación por VI muestra un efecto de 0,7 meses. Esta estimación muestra que los resultados son robustos a la restricción de la muestra a este subgrupo.

CUADRO No 5: $\quad$ EFECTO DE EDUCACIÓN TÉCNICO-PROFESIONAL EN NÚMERO DE MESES DE EMPLEO EN 2010

\begin{tabular}{|c|c|c|c|c|}
\hline $\begin{array}{l}\text { Variable dependiente: } \\
\text { número de meses de } \\
\text { empleo en } 2010\end{array}$ & $\begin{array}{l}\mathrm{MCO} \\
\text { todos }\end{array}$ & $\begin{array}{c}\text { MCO } \\
\text { excluyendo } \\
\text { a los } \\
\text { matriculados } \\
\text { en educación } \\
\text { terciaria entre } \\
2007-2010\end{array}$ & VI todos & $\begin{array}{c}\text { VI excluyendo } \\
\text { a los matricu- } \\
\text { lados en } \\
\text { educación } \\
\text { terciaria } \\
\text { entre } 2007 \text { - } \\
2010\end{array}$ \\
\hline $\mathrm{TP}$ & $\begin{array}{l}0,796 * * * \\
(0,0435)\end{array}$ & $\begin{array}{l}0,640 * * * \\
(0,0533)\end{array}$ & $\begin{array}{c}0,728 * * * \\
(0,215)\end{array}$ & $\begin{array}{c}0,744 * * * \\
(0,227)\end{array}$ \\
\hline SIMCE promedio 2001 & $\begin{array}{r}0,00260 * * * \\
(0,000449)\end{array}$ & $\begin{array}{r}0,00425^{* * *} \\
(0,000649)\end{array}$ & $\begin{array}{l}0,00253^{* * *} \\
(0,000504)\end{array}$ & $\begin{array}{l}0,00426 * * * \\
(0,000649)\end{array}$ \\
\hline Sexo & $\begin{array}{l}1,399 * * * \\
(0,0368)\end{array}$ & $\begin{array}{l}1,900 * * * \\
(0,0471)\end{array}$ & $\begin{array}{l}1,403 * * * \\
(0,0389)\end{array}$ & $\begin{array}{l}1,896 * * * \\
(0,0481)\end{array}$ \\
\hline $\begin{array}{l}\text { Existencia esc. madre } \times \\
\text { esc. madre }\end{array}$ & $\begin{array}{c}-0,0282 * * * \\
(0,00453)\end{array}$ & $\begin{array}{r}-0,00983^{*} \\
(0,00596)\end{array}$ & $\begin{array}{c}-0,0290^{* * *} \\
(0,00512)\end{array}$ & $\begin{array}{l}-0,00905 \\
(0,00619)\end{array}$ \\
\hline $\begin{array}{l}\text { Existencia esc. } \\
\text { madre }\end{array}$ & $\begin{array}{l}0,466 * * * \\
(0,0638)\end{array}$ & $\begin{array}{l}0,379 * * * \\
(0,0772)\end{array}$ & $\begin{array}{l}0,473 * * * \\
(0,0671)\end{array}$ & $\begin{array}{l}0,373 * * * \\
(0,0782)\end{array}$ \\
\hline Constante & $\begin{array}{c}4,480 * * * \\
(0,184)\end{array}$ & $\begin{array}{c}3,599 * * * \\
(0,243)\end{array}$ & $\begin{array}{c}4,532 * * * \\
(0,243)\end{array}$ & $\begin{array}{c}3,541 * * * \\
(0,272)\end{array}$ \\
\hline Test $\mathrm{F}$ primera etapa & & & 1030,66 & 875,83 \\
\hline $\mathrm{N}^{\circ}$ de observaciones & 72.747 & 44.858 & 72.747 & 44.858 \\
\hline
\end{tabular}

Nota: Sexo toma el valor 1 para hombres y 0 para mujeres. Existencia de escolaridad de la madre toma el valor 1 cuando contamos con el dato de esa escolaridad y 0 en caso contrario. Todas las especificaciones incluyen dummies regionales y dummies por tipo de dependencia del establecimiento de educación secundaria. Todas las regresiones excluyen a quienes están matriculados en educación terciaria en 2010 (véase texto para más detalles). Errores estándar entre paréntesis. * p $<0,1$, $* * \mathrm{p}<0,05 \mathrm{y} * * * \mathrm{p}<0,01$. 
Un posible problema relacionado con el uso del estimador de variables instrumentales es la existencia de lo que en la literatura se denomina "instrumentos débiles" (Staiger y Stock, 1997). En la presencia de este fenómeno puede existir un sesgo importante en la estimación. Una forma de determinar la presencia de este hecho es analizar el test de significancia conjunta de los parámetros ligados a las variables de exclusión $\left(\left\{\delta_{l j}\right\}_{j=1}^{S}\right.$ y $\left\{\delta_{2 j}\right\}_{j=1}^{S}$ en la ecuación 3). El Cuadro $N^{\circ} 5$ muestra los resultados de las pruebas estadísticas (test F) para la estimación de VI. Los resultados indican que en ambos casos los valores superan ampliamente 10, valor que usualmente es considerado como el mínimo valor para descartar la posibilidad de que estamos en presencia de instrumentos débiles.

Al desagregar el análisis entre los distintos tipos de educación TP vemos que existe una heterogeneidad importante en el efecto sobre el número de meses de empleo. El Cuadro $\mathrm{N}^{\circ} 6$ presenta estos resultados. La estimación por MCO sugiere que todas las categorías entregan mejores resultados en términos de empleabilidad que la educación $\mathrm{CH}$, con efectos que van entre 0,5 y 0,8 meses adicionales de empleo por año, dependiendo de la categoría de educación TP. El mismo cuadro presenta la estimación por VI, la que muestra un panorama similar, aunque la categoría Técnica tendría el mismo efecto sobre empleo que la educación CH. Dicha categoría incluye especialidades como Atención de Párvulos y Servicios de Turismo. Determinar la razón por la cual esta categoría y, en último término, sus especialidades, obtienen peores resultados en empleo formal, requiere un análisis detallado que escapa del objetivo de este trabajo. Adicionalmente, la estimación por VI amplía el rango de efectos estando ahora entre 0,5 y 1,6, que corresponden a las categorías Comercial e Industrial, respectivamente. Nuevamente, al excluir a quienes asistieron a educación terciaria entre 2007 y 2010 nuestros resultados no cambian significativamente. La estimación por MCO para esta submuestra es similar en magnitud a los efectos estimados por este método para la muestra completa. La única excepción es la categoría Marítima, la que no muestra un efecto significativamente diferente al de la educación $\mathrm{CH}$. Al corregir por endogeneidad mediante VI en nuestra submuestra el panorama es idéntico en signo y significancia al comentado antes usando VI para toda la muestra. La única diferencia está en que el tamaño de los efectos se reduce. Nuevamente la categoría Industrial es la que tiene el mayor efecto con 1,3 meses de empleo 
CUADRO N ${ }^{\circ}$ 6: EFECTO DE CADA CATEGORÍA DE EDUCACIÓN TÉCNICO-PROFESIONAL EN NÚMERO DE MESES DE EMPLEO EN 2010

\begin{tabular}{|c|c|c|c|c|}
\hline $\begin{array}{l}\text { Variable dependiente: } \\
\text { número de meses de } \\
\text { empleo en } 2010\end{array}$ & $\begin{array}{l}\mathrm{MCO} \\
\text { todos }\end{array}$ & $\begin{array}{c}\text { MCO } \\
\text { excluyendo } \\
\text { a los } \\
\text { matriculados } \\
\text { en educación } \\
\text { terciaria entre } \\
2007-2010\end{array}$ & VI todos & $\begin{array}{c}\text { VI excluyendo } \\
\text { a los matricu- } \\
\text { lados en } \\
\text { educación } \\
\text { terciaria } \\
\text { entre } 2007 \text { - } \\
2010\end{array}$ \\
\hline Comercial & $\begin{array}{c}0,791 * * * \\
(0,0534)\end{array}$ & $\begin{array}{c}0,655^{* * *} \\
(0,0659)\end{array}$ & $\begin{array}{c}0,493 * * * \\
(0,178)\end{array}$ & $\begin{array}{c}0,535 * * * \\
(0,201)\end{array}$ \\
\hline Industrial & $\begin{array}{c}0,862 * * * \\
(0,0615)\end{array}$ & $\begin{array}{c}0,579 * * * \\
(0,0729)\end{array}$ & $\begin{array}{c}1,581 * * * \\
(0,199)\end{array}$ & $\begin{array}{c}1,261 * * * \\
(0,223)\end{array}$ \\
\hline Técnico & $\begin{array}{c}0,535 * * * \\
(0,0787)\end{array}$ & $\begin{array}{c}0,496 * * * \\
(0,0884)\end{array}$ & $\begin{array}{c}-0,190^{* * *} \\
(0,288)\end{array}$ & $\begin{array}{c}-0,0602 * * * \\
(0,297)\end{array}$ \\
\hline Agrícola & $\begin{array}{c}0,556^{* * * *} \\
(0,105)\end{array}$ & $\begin{array}{l}0,360^{*} \\
(0,113)\end{array}$ & $\begin{array}{c}0,950^{* * * *} \\
(0,244)\end{array}$ & $\begin{array}{c}0,817 * * * \\
(0,266)\end{array}$ \\
\hline Marítimo & $\begin{array}{c}0,620^{* * * *} \\
(0,209)\end{array}$ & $\begin{array}{c}0,284 \\
(0,233)\end{array}$ & $\begin{array}{c}1,339 * * * \\
(0,438)\end{array}$ & $\begin{array}{c}0,928^{* * *} \\
(0,283)\end{array}$ \\
\hline Híbrido & $\begin{array}{l}0,530 * * * \\
(0,10369)\end{array}$ & $\begin{array}{c}0,394 * * * \\
(0,0452)\end{array}$ & $\begin{array}{c}0,387 * * * \\
(0,0787)\end{array}$ & $\begin{array}{c}0,283 * * * \\
(0,0926)\end{array}$ \\
\hline Simce promedio 2001 & $\begin{array}{c}0,00329 * * * \\
(0,000376)\end{array}$ & $\begin{array}{c}0,00505 * * * \\
(0,000512)\end{array}$ & $\begin{array}{c}0,00304 * * * \\
(0,000416)\end{array}$ & $\begin{array}{c}0,00485 * * * \\
(0,000543)\end{array}$ \\
\hline Sexo & $\begin{array}{l}1,620 * * * \\
(0,0311)\end{array}$ & $\begin{array}{l}2,094 * * * \\
(0,0382)\end{array}$ & $\begin{array}{l}1,433 * * * \\
(0,0489)\end{array}$ & $\begin{array}{l}1.910^{* * *} \\
(0.0610)\end{array}$ \\
\hline $\begin{array}{l}\text { Existencia esc. madre } \times \\
\text { esc. madre }\end{array}$ & $\begin{array}{c}-0,0261 * * * \\
(0,00367)\end{array}$ & $\begin{array}{c}-0,0120^{* * *} \\
(0,00460)\end{array}$ & $\begin{array}{c}-0,0274 * * * \\
(0,00381)\end{array}$ & $\begin{array}{c}-0,0123^{* * *} \\
(0,00466)\end{array}$ \\
\hline Existencia esc. madre & $\begin{array}{l}0,428 * * * \\
(0,0506)\end{array}$ & $\begin{array}{l}0,371 * * * \\
(0,0591)\end{array}$ & $\begin{array}{l}0,459 * * * \\
(0,0524)\end{array}$ & $\begin{array}{l}0,395^{* * *} \\
(0,0606)\end{array}$ \\
\hline Constante & $\begin{array}{c}4,257 * * * \\
(0,137)\end{array}$ & $\begin{array}{c}3,544 * * * \\
(0,170)\end{array}$ & $\begin{array}{c}4,505^{* * *} \\
(0,169)\end{array}$ & $\begin{array}{c}3,741^{* * *} \\
(0,195)\end{array}$ \\
\hline $\mathrm{N}^{\circ}$ de observaciones & 113.152 & 76.605 & 113.152 & 76.605 \\
\hline
\end{tabular}

Nota: Sexo toma el valor 1 para hombres y 0 para mujeres. Existencia de escolaridad de la madre toma el valor 1 cuando contamos con el dato de esa escolaridad y 0 en caso contrario. Todas las especificaciones incluyen dummies regionales y dummies por tipo de dependencia del establecimiento de educación secundaria. Todas las regresiones excluyen a quienes están matriculados en educación terciaria en 2010 (véase texto para más detalles). Errores estándar entre paréntesis. ${ }^{*} \mathrm{p}<0,1$, $* * \mathrm{p}<0,05 \mathrm{y} * * * \mathrm{p}<0,01$. 
adicional por año. El Cuadro $\mathrm{N}^{\circ} 7$ presenta los tests $\mathrm{F}$ del test de significancia conjunta asociado a los instrumentos en cada una de las primeras etapas de la estimación por variables instrumentales. Vemos que todos los tests realizados están por sobre 600 , lo que sugiere que no estamos en presencia de instrumentos débiles en ninguna de las estimaciones por VI presentadas en este trabajo.

Hasta ahora hemos establecido la ventaja de la educación TP en términos de empleo formal. Sin embargo, esto no necesariamente se traducirá en una mejora monetaria si el salario promedio que reciben es significativamente menor al de los egresados de $\mathrm{CH}$. Es por ello que analizamos el impacto que tiene la educación TP sobre los salarios que los individuos obtienen en 2010.

Tal como mencionamos en la sección 4, las diferencias de salarios existentes entre las modalidades de educación secundaria dependen de cómo se defina el salario anual promedio. Es por esto que presentamos el efecto de la educación TP sobre salarios utilizando las dos definiciones que hemos introducido en las secciones 4 y 5 .

\begin{tabular}{|c|c|c|c|c|c|c|}
\hline \multirow[t]{3}{*}{ CUADRO $\mathrm{N}^{\circ}$} & \multicolumn{6}{|c|}{$\begin{array}{l}\text { TEST F PRIMERA ETAPA DE VARIABLES INSTRUMENTALES PARA } \\
\text { LAS ESPECIFICACIONES QUE CONSIDERAN LAS CATEGORÍAS DE } \\
\text { TP POR SEPARADO }\end{array}$} \\
\hline & \multicolumn{2}{|c|}{ Resultados empleo } & \multicolumn{2}{|c|}{$\begin{array}{c}\text { Resultados salario } \\
\text { promedio }\end{array}$} & \multicolumn{2}{|c|}{$\begin{array}{l}\text { Resultados salario } \\
\text { promedio meses } \\
\text { empleo }\end{array}$} \\
\hline & IV todos & $\begin{array}{c}\text { VI sin } \\
\text { matricula- } \\
\text { dos educ. } \\
\text { terciaria }\end{array}$ & IV todos & $\begin{array}{c}\text { VI sin } \\
\text { matricula- } \\
\text { dos educ. } \\
\text { terciaria }\end{array}$ & IV todos & $\begin{array}{c}\text { VI sin } \\
\text { matricula- } \\
\text { dos educ. } \\
\text { terciaria }\end{array}$ \\
\hline Comercial & 916,51 & 713,47 & 916,51 & 713,47 & 679,48 & 526,34 \\
\hline Industrial & $1.057,02$ & 838,95 & $1.057,02$ & 838,95 & 884,40 & 706,13 \\
\hline Técnico & 793,39 & 648,28 & 793,39 & 648,28 & 565,58 & 457,53 \\
\hline Agrícola & $2.361,35$ & $1.655,68$ & $2.361,35$ & $1.655,68$ & $1.867,09$ & $1.298,60$ \\
\hline Marítimo & $2.683,34$ & $2.077,42$ & $2.683,34$ & $2.077,42$ & $2.201,61$ & $1.705,85$ \\
\hline Híbrido & $3.641,02$ & $2.731,21$ & $3.641,02$ & $2.731,21$ & $2.742,99$ & $2.060,34$ \\
\hline
\end{tabular}

Nota: Los tests F tienen como hipótesis nula que los coeficientes asociados a las variables de exclusión son iguales a 0 en la primera etapa. Todas las regresiones de la primera etapa consideran las mismas variables de control definidas en el texto: Simce promedio 2001, sexo, escolaridad de la madre, presencia de la madre, dummies regionales y dummies de dependencia de establecimiento de educación secundaria. 
Primero analizamos los resultados al considerar el salario promedio que los individuos obtienen en los 12 meses del 2010 independientemente de si están empleados. Así, consideramos salarios iguales a 0 para quienes no están empleados en el sector formal en un determinado mes del año. Esta definición nos permite analizar el bienestar material que en un año aporta el tipo de educación secundaria que el individuo cursó. El Cuadro $N^{\circ} 8$ muestra la estimación utilizando la educación TP agregada, donde notamos que la estimación por MCO sugiere un efecto positivo de $\$ 20.600$ pesos mensuales, resultado que se revierte

CUADRO N 8: $\quad$ EFECTO DE LA EDUCACIÓN TÉCNICO-PROFESIONAL EN SALARIO PROMEDIO 2010

\begin{tabular}{|c|c|c|c|c|}
\hline $\begin{array}{l}\text { Variable dependiente: } \\
\text { salario promedio en } 2010\end{array}$ & $\begin{array}{l}\mathrm{MCO} \\
\text { todos }\end{array}$ & $\begin{array}{c}\text { MCO } \\
\text { excluyendo } \\
\text { a los } \\
\text { matriculados } \\
\text { en educación } \\
\text { terciaria entre } \\
2007-2010\end{array}$ & VI todos & $\begin{array}{c}\text { VI excluyendo } \\
\text { a los matricu- } \\
\text { lados en } \\
\text { educación } \\
\text { terciaria } \\
\text { entre } 2007 \text { - } \\
2010\end{array}$ \\
\hline TP & $\begin{array}{c}20.600^{* * *} \\
(1.945)\end{array}$ & $\begin{array}{c}24.182 * * * \\
(1.982)\end{array}$ & $\begin{array}{l}-28.738^{* * *} \\
(9.673)\end{array}$ & $\begin{array}{c}5.780 \\
(8.428)\end{array}$ \\
\hline SIMCE promedio 2001 & $\begin{array}{c}833,4 * * * \\
(20,10)\end{array}$ & $\begin{array}{c}477,6^{* * * *} \\
(24,11)\end{array}$ & $\begin{array}{c}780,0^{* * *} \\
(22,64)\end{array}$ & $\begin{array}{c}477,0^{* * *} \\
(24,13)\end{array}$ \\
\hline Sexo & $\begin{array}{c}70.639 * * * \\
(1.647)\end{array}$ & $\begin{array}{c}90.269 * * * \\
(1.750)\end{array}$ & $\begin{array}{c}73.599 * * * \\
(1.749)\end{array}$ & $\begin{array}{l}91.103 * * * \\
(1.790)\end{array}$ \\
\hline $\begin{array}{l}\text { Existencia esc. madre } \times \\
\text { esc. madre }\end{array}$ & $\begin{array}{c}322,9 \\
(202,7)\end{array}$ & $\begin{array}{c}362,3 \\
(221,6)\end{array}$ & $\begin{array}{l}-237,8 \\
(230,3)\end{array}$ & $\begin{array}{c}223,7 \\
(230,2)\end{array}$ \\
\hline Existencia esc. madre & $\begin{array}{l}5.802 * * * \\
(2.855)\end{array}$ & $\begin{array}{l}7.186^{* * * *} \\
(2.870)\end{array}$ & $\begin{array}{c}10,678^{* * *} \\
(3.016)\end{array}$ & $\begin{array}{c}8.216^{* * *} \\
(2.908)\end{array}$ \\
\hline Constante & $\begin{array}{c}-56.301 * * * \\
(8.238)\end{array}$ & $\begin{array}{r}-11.277 \\
(9.020)\end{array}$ & $\begin{array}{r}-19.095^{*} \\
(10.931)\end{array}$ & $\begin{array}{l}-1.031 \\
(10.113)\end{array}$ \\
\hline Test $\mathrm{F}$ primera etapa & & & 1030,66 & 875,83 \\
\hline $\mathrm{N}^{\circ}$ de observaciones & 72.747 & 44.858 & 72.747 & 44.858 \\
\hline
\end{tabular}

Nota: Sexo toma el valor 1 para hombres y 0 para mujeres. Existencia de escolaridad de la madre toma el valor 1 cuando contamos con el dato de esa escolaridad y 0 en caso contrario. Todas las especificaciones incluyen dummies regionales y dummies por tipo de dependencia del establecimiento de educación secundaria. Todas las regresiones excluyen a quienes están matriculados en educación terciaria en 2010 (véase texto para más detalles). Errores estándar entre paréntesis. * p $<0,1$, $* * \mathrm{p}<0,05 \mathrm{y} * * * \mathrm{p}<0,01$. 
al utilizar la estimación de variables instrumentales. En efecto, luego de corregir por sesgo de selección, la educación TP tiene un efecto negativo sobre el salario anual. La reducción mensual en el salario por haber estudiado en un colegio TP y no en uno $\mathrm{CH}$ es de 28.738 pesos. Asimismo, al considerar solo a quienes no cursaron educación terciaria entre 2007-2010, la estimación por MCO nuevamente muestra un efecto positivo y significativo de 24.182 pesos mensuales, pero los resultados de nuevo cambian al utilizar el estimador de VI. En este caso, si bien el retorno es positivo, este no es estadísticamente significativo. Como vemos, corregir la endogeneidad en el tipo de educación media es fundamental, pues no hacerlo puede llevar a resultados y conclusiones totalmente diferentes.

Por otro lado, nuestros resultados muestran que en este margen existen diferencias importantes entre las distintas categorías de educación TP. En efecto, el Cuadro $\mathrm{N}^{\circ} 9$ muestra la estimación por MCO y VI al desagregar por tipo de educación media. Ambos estimadores muestran diferencias importantes en los retornos entre los distintos tipos de educación TP. En efecto, el estimador de MCO muestra un retorno de \$39.058 pesos mensuales para la categoría industrial, en tanto muestra un efecto promedio negativo de $\$ 7.368$ asociado a haber estudiado una especialidad de la categoría técnica. Dicha relación se mantiene al corregir por selección, aunque los retornos son ahora $\$ 42.100$ y $-\$ 54.610$ para las categorías Industrial y Técnica, respectivamente. Además, la estimación por VI muestra que tanto las categorías Comercial como Agrícola obtienen salarios mensuales que no son significativamente diferentes de los de la educación CH. Esto muestra que la educación TP en Chile presenta una importante heterogeneidad en los resultados laborales de sus egresados, pues si bien las categorías antes mencionadas son competitivas e incluso obtienen mejores resultados en dos casos, tenemos también la categoría técnica cuyos egresados obtienen mensualmente 54.610 pesos menos que sus pares de liceos $\mathrm{CH}$.

Resulta interesante además constatar que esta heterogeneidad se mantiene al analizar al grupo de individuos que no asistió a educación terciaria entre 2007 y 2010. En general, al reducir la muestra a este grupo aumentan los retornos de todos los tipos de educación TP. Esto demuestra el mayor acceso al sistema de educación superior de los egresados de $\mathrm{CH}$, y explica, por lo menos en parte, nuestros resultados previos. 
CUADRO N ${ }^{\circ}$ 9: $\quad$ EFECTO DE CADA CATEGORÍA DE EDUCACIÓN TÉCNICO-PROFESIONAL EN SALARIO PROMEDIO EN 2010

\begin{tabular}{|c|c|c|c|c|}
\hline $\begin{array}{l}\text { Variable dependiente: } \\
\text { salario promedio en } 2010\end{array}$ & $\begin{array}{l}\mathrm{MCO} \\
\text { todos }\end{array}$ & $\begin{array}{c}\text { MCO } \\
\text { excluyendo } \\
\text { a los } \\
\text { matriculados } \\
\text { en educación } \\
\text { terciaria entre } \\
2007-2010\end{array}$ & VI todos & $\begin{array}{l}\text { VI excluyendo } \\
\text { a los matricu- } \\
\text { lados en } \\
\text { educación } \\
\text { terciaria } \\
\text { entre } 2007 \text { - } \\
2010\end{array}$ \\
\hline Comercial & $\begin{array}{c}15.639 * * * \\
(2.284)\end{array}$ & $\begin{array}{c}21.546^{* * *} \\
(2.429)\end{array}$ & $\begin{array}{c}-9.793 * * * \\
(7.599)\end{array}$ & $\begin{array}{c}8.057 \\
(7.395)\end{array}$ \\
\hline Industrial & $\begin{array}{c}39.058^{* * *} \\
(2.633)\end{array}$ & $\begin{array}{c}38.311^{* * *} \\
(2.687)\end{array}$ & $\begin{array}{c}42.100^{* * *} \\
(8.521)\end{array}$ & $\begin{array}{c}56.641 * * * \\
(8.227)\end{array}$ \\
\hline Técnico & $\begin{array}{c}-7368 * * * \\
(3.367)\end{array}$ & $\begin{array}{c}1.265^{* * *} \\
(3.259)\end{array}$ & $\begin{array}{c}-54.610^{* * *} \\
(12.310)\end{array}$ & $\begin{array}{c}-23.894 * * * \\
(10.963)\end{array}$ \\
\hline Agrícola & $\begin{array}{l}-3.269 \\
(4.474)\end{array}$ & $\begin{array}{c}807,2 \\
(4.173)\end{array}$ & $\begin{array}{l}-12.234 \\
(10.439)\end{array}$ & $\begin{array}{l}6.278 \\
(9.790)\end{array}$ \\
\hline Marítimo & $\begin{array}{c}20.288 * * * \\
(8.941)\end{array}$ & $\begin{array}{c}21.300^{* *} \\
(8.595)\end{array}$ & $\begin{array}{l}35.982 * \\
(18.729)\end{array}$ & $\begin{array}{c}56.963^{* * *} \\
(17.182)\end{array}$ \\
\hline Híbrido & $\begin{array}{c}8.260 * * * \\
(1.580)\end{array}$ & $\begin{array}{c}11.547 * * * \\
(1.665)\end{array}$ & $\begin{array}{c}-8.023 * * \\
(3.367)\end{array}$ & $\begin{array}{c}2.601 \\
(3.412)\end{array}$ \\
\hline Simce promedio 2001 & $\begin{array}{c}761,5^{* * *} \\
(16,09)\end{array}$ & $\begin{array}{c}449,9 * * * \\
(18,86)\end{array}$ & $\begin{array}{c}728,9 * * * \\
(17,81)\end{array}$ & $\begin{array}{c}441,7 * * * \\
(20,00)\end{array}$ \\
\hline Sexo & $\begin{array}{c}75.888^{* * *} \\
(1.331)\end{array}$ & $\begin{array}{c}93.915^{* * *} \\
(1.409)\end{array}$ & $\begin{array}{c}70.706^{* * *} \\
(2.092)\end{array}$ & $\begin{array}{c}87.405^{* * *} \\
(2.247)\end{array}$ \\
\hline $\begin{array}{l}\text { Existencia esc. madre } \times \\
\text { esc. madre }\end{array}$ & $\begin{array}{c}325,2 * * \\
(157,0\end{array}$ & $\begin{array}{l}312,6^{*} \\
(169,6)\end{array}$ & $\begin{array}{c}101,5 \\
(163,2)\end{array}$ & $\begin{array}{c}256,9 \\
(171,6)\end{array}$ \\
\hline Existencia esc. madre & $\begin{array}{c}3.473 \\
(2.164)\end{array}$ & $\begin{array}{l}4.497^{* *} \\
(2.179)\end{array}$ & $\begin{array}{c}5.896^{* * *} \\
(2.243)\end{array}$ & $\begin{array}{l}5.694 * * \\
(2.234)\end{array}$ \\
\hline Constante & $\begin{array}{c}-28.881 * * * \\
(5.861)\end{array}$ & $\begin{array}{c}13.544 * * \\
(6.252)\end{array}$ & $\begin{array}{l}-5.505 \\
(7.222)\end{array}$ & $\begin{array}{c}24.673 * * * \\
(7.189)\end{array}$ \\
\hline $\mathrm{N}^{\circ}$ de observaciones & 113.152 & 76.605 & 113.152 & 76.605 \\
\hline
\end{tabular}

Nota: Sexo toma el valor 1 para hombres y 0 para mujeres. Existencia de escolaridad de la madre toma el valor 1 cuando contamos con el dato de esa escolaridad y 0 en caso contrario. Todas las especificaciones incluyen dummies regionales y dummies por tipo de dependencia del establecimiento de educación secundaria. Todas las regresiones excluyen a quienes están matriculados en educación terciaria en 2010 (véase texto para más detalles). Errores estándar entre paréntesis. ${ }^{*} \mathrm{p}<0,1$, $* * \mathrm{p}<0,05 \mathrm{y} * * * \mathrm{p}<0,01$. 
Una pregunta más exigente es si las categorías de educación TP son competitivas al considerar el salario promedio solo en los meses en que los individuos cuentan con empleo formal. Esta es una pregunta diferente a las que hemos abordado hasta ahora, pues apunta al nivel de salario promedio que los egresados de cada tipo de educación secundaria obtendrían si es que encuentran trabajo en el sector formal, cuestión que como sabemos no siempre ocurre y que difiere entre categorías (véase Figura $\mathrm{N}^{\circ}$ 5). Tal como vemos en el Cuadro $\mathrm{N}^{\circ} 10$, sin corregir por selección, los resultados sugieren que la educación TP genera un salario mensual en meses de empleo que no es significativamente dis-

CUADRO N ${ }^{\circ}$ 10: EFECTO DE LA EDUCACIÓN TÉCNICO-PROFESIONAL EN SALARIO PROMEDIO EN MESES DE EMPLEO EN 2010

\begin{tabular}{|c|c|c|c|c|}
\hline $\begin{array}{l}\text { Variable dependiente: } \\
\text { salario promedio en } \\
\text { meses de empleo en } 2010\end{array}$ & $\begin{array}{l}\mathrm{MCO} \\
\text { todos }\end{array}$ & $\begin{array}{c}\text { MCO } \\
\text { excluyendo } \\
\text { a los } \\
\text { matriculados } \\
\text { en educación } \\
\text { terciaria entre } \\
2007-2010\end{array}$ & VI todos & $\begin{array}{l}\text { VI excluyendo } \\
\text { a los matricu- } \\
\text { lados en } \\
\text { educación } \\
\text { terciaria } \\
\text { entre 2007- } \\
2010\end{array}$ \\
\hline TP & $\begin{array}{l}-1896 \\
(2.117)\end{array}$ & $\begin{array}{c}14.941 * * * \\
(2.060)\end{array}$ & $\begin{array}{c}-81.390 * * * \\
(10.595)\end{array}$ & $\begin{array}{l}-13.925 \\
(8.756)\end{array}$ \\
\hline SIMCE promedio 2001 & $\begin{array}{c}1.361 * * * \\
(22,06)\end{array}$ & $\begin{array}{c}651,4 * * * \\
(25,10)\end{array}$ & $\begin{array}{c}1.280^{* * *} \\
(24,73)\end{array}$ & $\begin{array}{c}653,5^{* * * *} \\
(25,18)\end{array}$ \\
\hline Sexo & $\begin{array}{c}62.481 * * * \\
(1.810)\end{array}$ & $\begin{array}{c}79.584 * * * \\
(1.830)\end{array}$ & $\begin{array}{c}67671 * * * \\
(1.954)\end{array}$ & $\begin{array}{c}80.937 * * * \\
(1.878)\end{array}$ \\
\hline $\begin{array}{l}\text { Existencia esc. madre } \times \\
\text { esc. madre }\end{array}$ & $\begin{array}{c}2.173 * * * \\
(221,9)\end{array}$ & $\begin{array}{c}1.292 * * * \\
(228,8)\end{array}$ & $\begin{array}{c}1.262 * * * \\
(254,3)\end{array}$ & $\begin{array}{c}1.081 * * * \\
(237,7)\end{array}$ \\
\hline Existencia esc. madre & $\begin{array}{c}-13.800^{* * *} \\
\quad(3.119)\end{array}$ & $\begin{array}{l}-5.175^{*} \\
(2.971)\end{array}$ & $\begin{array}{l}-6.058^{*} \\
(3.316)\end{array}$ & $\begin{array}{l}-3651 \\
(3.013)\end{array}$ \\
\hline Constante & $\begin{array}{c}-33.889 * * * \\
(9.154)\end{array}$ & $\begin{array}{l}80.066 * * * \\
(9.573)\end{array}$ & $\begin{array}{c}25.778 * * \\
(12.107)\end{array}$ & $\begin{array}{c}95.785 * * * \\
(10.658)\end{array}$ \\
\hline Test $F$ primera etapa & & & 773,97 & 654,97 \\
\hline $\mathrm{N}^{\circ}$ de observaciones & 54.501 & 33.365 & 54.401 & 33.365 \\
\hline
\end{tabular}

Nota: Sexo toma el valor 1 para hombres y 0 para mujeres. Existencia de escolaridad de la madre toma el valor 1 cuando contamos con el dato de esa escolaridad y 0 en caso contrario. Todas las especificaciones incluyen dummies regionales y dummies por tipo de dependencia del establecimiento de educación secundaria. Todas las regresiones excluyen a quienes están matriculados en educación terciaria en 2010 (véase texto para más detalles). Errores estándar entre paréntesis. ${ }^{*} \mathrm{p}<0,1$, $* * \mathrm{p}<0,05 \mathrm{y} * * * \mathrm{p}<0,01$. 
tinto al que obtienen los egresados de la educación $\mathrm{CH}$. Sin embargo, al corregir por selección utilizando variables instrumentales, vemos que el efecto promedio sobre los salarios de estudiar en la educación TP es de -81.390 pesos. Al restringir la muestra a quienes no cursaron estudios terciarios entre 2007 y 2010, encontramos nuevamente que el retorno de la educación TP es positivo cuando no se corrige por selección, pero no es significativo al estimarlo a través de VI. Esto sugiere que en esta submuestra el retorno de la educación TP no es significativamente diferente al de la educación $\mathrm{CH}$.

Como ya hemos hecho notar, considerar la educación TP de manera agregada no permite analizar la heterogeneidad existente entre sus categorías. El Cuadro $\mathrm{N}^{\circ} 11$ muestra el efecto de la educación TP en sus distintas categorías sobre el salario promedio en los meses de empleo en 2010. En primera instancia, al analizar los resultados por MCO, vemos que la mayoría de las categorías de educación TP tienen asociado un retorno negativo. Las 20 únicas excepciones serían la educación Industrial y Marítima que tendrían retornos de 17.305 pesos mensuales y no significativamente distinto al de $\mathrm{CH}$, respectivamente. Por otro lado, al corregir por sesgo de selección, vemos que no existe categoría alguna en que la educación TP tenga mayor retorno que la educación $\mathrm{CH}$. Las únicas categorías que obtienen salarios que no son significativamente diferentes al de la modalidad $\mathrm{CH}$ son las categorías Industrial y Marítima. Este es sin duda el margen en que peor desempeño muestran los egresados de colegios TP, aunque nuevamente hay dos categorías que nuestros resultados sugieren que no son diferentes a la modalidad $\mathrm{CH}$ en términos de salarios en meses de empleo.

Si restringimos el análisis a quienes no se han matriculado en educación terciaria entre 2007 y 2010, vemos que la estimación por MCO sugiere varias categorías con retorno positivo: Comercial, Industrial, Marítima e Híbrida. Al corregir por sesgo de selección, esta situación se mantiene para dos categorías. En efecto, las categorías Industrial y Marítima presentan retorno positivo para este subgrupo, pues generan un salario de 33.198 y 56.535 pesos mensuales, respectivamente. Nuestra evidencia permite concluir que la educación TP aumenta el número de meses de empleo formal, aunque el panorama es mixto cuando analizamos los ingresos laborales.

Los resultados más auspiciosos en términos de ingresos los encontramos entre aquellos individuos que no aparecen asistiendo al 
CUADRO N 11: $\quad$ EFECTO DE CADA CATEGORÍA DE EDUCACIÓN TÉCNICO-PROFESIONAL EN SALARIO PROMEDIO EN MESES DE EMPLEO EN 2010

\begin{tabular}{|c|c|c|c|c|}
\hline $\begin{array}{l}\text { Variable dependiente: } \\
\text { salario promedio en meses } \\
\text { de empleo en } 2010\end{array}$ & $\begin{array}{l}\mathrm{MCO} \\
\text { todos }\end{array}$ & $\begin{array}{c}\text { MCO } \\
\text { excluyendo } \\
\text { a los } \\
\text { matriculados } \\
\text { en educación } \\
\text { terciaria entre } \\
2007-2010\end{array}$ & VI todos & $\begin{array}{c}\text { VI excluyendo } \\
\text { a los matricu- } \\
\text { lados en } \\
\text { educación } \\
\text { terciaria } \\
\text { entre } 2007 \text { - } \\
2010\end{array}$ \\
\hline Comercial & $\begin{array}{c}-8.764 * * * \\
(2.456)\end{array}$ & $\begin{array}{c}12.132 * * * \\
(2.517)\end{array}$ & $\begin{array}{c}-31.143 * * * \\
(8.235)\end{array}$ & $\begin{array}{l}-2.324 \\
(7.706)\end{array}$ \\
\hline Industrial & $\begin{array}{c}17.305 * * * \\
(2.727)\end{array}$ & $\begin{array}{c}31.397^{* * *} \\
(2.664)\end{array}$ & $\begin{array}{l}-3.991 \\
(8.565)\end{array}$ & $\begin{array}{c}33.198 * * * \\
(7.900)\end{array}$ \\
\hline Técnico & $\begin{array}{c}-33.135 * * * \\
(3.727)\end{array}$ & $\begin{array}{c}-13.636^{* * *} \\
(3.469)\end{array}$ & $\begin{array}{l}-85.873 \\
(13.937)\end{array}$ & $\begin{array}{c}-39.085^{* * *} \\
(11.917)\end{array}$ \\
\hline Agrícola & $\begin{array}{c}-28.403 * * * \\
(4.744)\end{array}$ & $\begin{array}{c}-10.469^{* *} \\
(4.223)\end{array}$ & $\begin{array}{c}-54.686^{* * * *} \\
(10.943)\end{array}$ & $\begin{array}{l}-19.935^{* *} \\
(9.819)\end{array}$ \\
\hline Marítimo & $\begin{array}{c}849,3 \\
(9.321)\end{array}$ & $\begin{array}{c}21.162 * * \\
(8.540)\end{array}$ & $\begin{array}{c}5.838 \\
(18.967)\end{array}$ & $\begin{array}{c}56.535^{* * *} \\
(16.574)\end{array}$ \\
\hline Híbrido & $\begin{array}{l}-9.938 * * * \\
(1.704)\end{array}$ & $\begin{array}{r}3.462 * * \\
(1.723)\end{array}$ & $\begin{array}{c}-32.410^{* * * *} \\
(3.678)\end{array}$ & $\begin{array}{l}-9-519 * * * \\
(3.564)\end{array}$ \\
\hline Simce promedio 2001 & $\begin{array}{c}1.193 * * * \\
(17,33)\end{array}$ & $\begin{array}{c}571,2 * * * \\
(19,39)\end{array}$ & $\begin{array}{c}1.146^{* * *} \\
(19,24)\end{array}$ & $\begin{array}{c}559,8^{* * *} \\
(20,66)\end{array}$ \\
\hline Sexo & $\begin{array}{c}63.094 * * * \\
(1.442)\end{array}$ & $\begin{array}{c}78.556^{* * *} \\
(1.465)\end{array}$ & $\begin{array}{c}61.865^{* * *} \\
(2.272)\end{array}$ & $\begin{array}{c}74.923 * * * \\
(2.330)\end{array}$ \\
\hline $\begin{array}{l}\text { Existencia esc. madre } \times \\
\text { esc. madre }\end{array}$ & $\begin{array}{c}1.937 * * * \\
(168,6)\end{array}$ & $\begin{array}{c}1.177 * * * \\
(173,4)\end{array}$ & $\begin{array}{c}1.628 * * * \\
(175,3)\end{array}$ & $\begin{array}{c}1.082 * * * \\
(175,4)\end{array}$ \\
\hline Existencia esc. madre & $\begin{array}{c}-14.185^{* * *} \\
(2.323)\end{array}$ & $\begin{array}{c}-7.781 * * * \\
(2.235)\end{array}$ & $\begin{array}{c}-11.586 * * * \\
(2.405)\end{array}$ & $\begin{array}{c}-6.752 * * * \\
(2.289)\end{array}$ \\
\hline Constante & $\begin{array}{c}25.678^{* * *} \\
(6.393)\end{array}$ & $\begin{array}{c}125.174 * * * \\
(6.531)\end{array}$ & $\begin{array}{c}54.472 * * * \\
(7.895)\end{array}$ & $\begin{array}{c}138.369 * * * \\
(7.516)\end{array}$ \\
\hline $\mathrm{N}^{\circ}$ de observaciones & 85.255 & 57.406 & 85.255 & 57.406 \\
\hline
\end{tabular}

Nota: Sexo toma el valor 1 para hombres y 0 para mujeres. Existencia de escolaridad de la madre toma el valor 1 cuando contamos con el dato de esa escolaridad y 0 en caso contrario. Todas las especificaciones incluyen dummies regionales y dummies por tipo de dependencia del establecimiento de educación secundaria. Todas las regresiones excluyen a quienes están matriculados en educación terciaria en 2010 (véase texto para más detalles). Errores estándar entre paréntesis. ${ }^{*} \mathrm{p}<0,1$, $* * \mathrm{p}<0,05 \mathrm{y} * * * \mathrm{p}<0,01$. 
sistema de educación superior durante el período 2007-2010 y cuando analizamos el ingreso promedio anual (incluyendo meses de desempleo, inactividad, empleo informal o empleo independiente). La heterogeneidad que reportamos entre las categorías representa un serio desafío desde el punto de vista de políticas públicas.

\section{Conclusiones}

En este trabajo presentamos la primera evidencia causal sobre el desempeño laboral de los egresados de los distintos tipos de educación media en Chile. Nuestro análisis se circunscribe a efectos sobre número de meses de empleo y salarios promedios mensuales.

Utilizamos una base de datos única, que reúne información sobre el tipo de educación en que participaba cada persona en segundo año medio en el año 2001 y remuneraciones durante 2010. Haciendo uso de variables de oferta de los diferentes tipos de educación instrumentalizamos la decisión de participación en cada tipo de educación para corregir la endogeneidad que presenta esta decisión. Esta estrategia ha sido ampliamente utilizada en la literatura (Card, 2001).

Nuestros resultados indican que la educación TP genera mayor empleabilidad aunque su efecto no pasa más allá de 0,7 meses anuales cuando consideramos agregadamente este tipo de educación. Cuando consideramos las categorías de educación TP por separado, el efecto alcanza 1,6 meses de empleo más al año para quienes estudiaron educación TP Industrial. Este es uno de los primeros datos que deberían estar disponibles para los jóvenes y sus familias a la hora de decidir qué tipo de educación secundaria escoger.

Este estudio muestra además que cuando consideramos la educación TP de manera agregada, esta tiene un efecto negativo sobre salarios, sin importar si consideramos el salario promedio de los meses de trabajo o de los 12 meses del año. Sin embargo, cuando consideramos solo a quienes no cursaron estudios terciarios entre 2007 y 2010, vemos que la educación TP tiene efecto positivo o nulo sobre salarios.

Dichos resultados esconden un nivel importante de heterogeneidad entre las distintas categorías de educación TP. En efecto, cuando consideramos separadamente el efecto de cada categoría de educación TP vemos por un lado que la categoría TP Industrial presenta efectos positivos sobre el salario promedio en 12 meses $(\$ 42.100)$ y un efecto 
nulo en el salario promedio en los meses de trabajo. Por otro lado, la categoría TP Técnica presenta efecto negativo bajo ambas definiciones de salario promedio ( $-\$ 54.610$ y $-\$ 85.873$ al considerar el salario promedio de 12 meses o el salario promedio en los meses de empleo, respectivamente). Esta heterogeneidad en el efecto de cada categoría de educación TP es importante a la hora de pensar en futuras reformas a las especialidades que cada categoría imparte y al pensar en cuáles son las categorías de educación TP que requieren mayor financiamiento.

En conjunto, la evidencia que hemos presentado plantea una visión optimista sobre el desarrollo de la educación media TP en Chile. Los jóvenes que hace una década cursaron este tipo de educación parecen tener mejores herramientas para enfrentar el mundo laboral en comparación con sus pares egresados de educación $\mathrm{CH}$. Sin embargo, nuestros resultados también alertan sobre las diferencias internas que deben ser abordadas y sobre la pertinencia de generar sistemas de información pública que permitan tomar mejores decisiones a los alumnos y sus familias. 


\section{Apéndice}

CUADRO A: NÚMERO DE MATRICULADOS POR TIPO DE EDUCACIÓN TP Y COMUNA EN EL AÑO 2000

\begin{tabular}{|c|c|c|c|c|c|}
\hline Comuna & Comercial & Industrial & Técnico & Agrícola & Marítimo \\
\hline Algarrobo & 309 & 0 & 28 & 0 & 0 \\
\hline Alhué & 0 & 0 & 0 & 0 & 0 \\
\hline Alto del Carmen & 0 & 0 & 0 & 0 & 0 \\
\hline Ancud & 701 & 213 & 128 & 113 & 76 \\
\hline Andacollo & 144 & 0 & 0 & 0 & 0 \\
\hline Angol & 1.285 & 564 & 699 & 225 & 0 \\
\hline Antártica & 0 & 0 & 0 & 0 & 0 \\
\hline Antofagasta & 2.893 & 1.861 & 1.589 & 0 & 0 \\
\hline Antuco & 0 & 0 & 0 & 0 & 0 \\
\hline Arauco & 188 & 272 & 0 & 0 & 0 \\
\hline Arica & 3.642 & 1.699 & 900 & 132 & 0 \\
\hline Aysén & 232 & 228 & 0 & 0 & 109 \\
\hline Buin & 823 & 270 & 158 & 221 & 0 \\
\hline Bulnes & 304 & 0 & 0 & 0 & 0 \\
\hline Cabildo & 0 & 222 & 0 & 0 & 0 \\
\hline Cabrero & 275 & 457 & 116 & 0 & 0 \\
\hline Calama & 1.463 & 1.516 & 264 & 0 & 0 \\
\hline Calbuco & 0 & 99 & 103 & 0 & 115 \\
\hline Caldera & 0 & 174 & 0 & 0 & 326 \\
\hline Calera & 774 & 1.063 & 26 & 0 & 0 \\
\hline Calera de Tango & 0 & 0 & 0 & 0 & 0 \\
\hline Calle Larga & 0 & 0 & 0 & 0 & 349 \\
\hline Camarones & 0 & 0 & 0 & 0 & 0 \\
\hline Camiña & 0 & 0 & 0 & 0 & 0 \\
\hline Canela & 0 & 0 & 0 & 103 & 0 \\
\hline Cañete & 326 & 0 & 0 & 101 & 0 \\
\hline Carahue & 617 & 120 & 0 & 0 & 0 \\
\hline Cartagena & 0 & 0 & 118 & 0 & 194 \\
\hline Casablanca & 0 & 287 & 45 & 0 & 0 \\
\hline Castro & 659 & 609 & 60 & 272 & 0 \\
\hline Catemu & 17 & 0 & 0 & 223 & 0 \\
\hline Cauquenes & 458 & 535 & 141 & 0 & 0 \\
\hline Cerrillos & 430 & 2.816 & 183 & 0 & 0 \\
\hline Cerro Navia & 1.407 & 321 & 99 & 0 & 0 \\
\hline Chaitén & 0 & 0 & 0 & 15 & 0 \\
\hline Chañaral & 408 & 218 & 0 & 0 & 0 \\
\hline Chanco & 0 & 0 & 0 & 35 & 0 \\
\hline Chépica & 115 & 0 & 0 & 0 & 0 \\
\hline
\end{tabular}




\begin{tabular}{|c|c|c|c|c|c|}
\hline Сотипа & Comercial & Industrial & Técnico & Agrícola & Marítimo \\
\hline Chiguayante & 0 & 136 & 0 & 0 & 0 \\
\hline Chile Chico & 0 & 0 & 86 & 0 & 0 \\
\hline Chillán & 3.790 & 1.131 & 748 & 461 & 0 \\
\hline Chillán Viejo & 0 & 0 & 0 & 0 & 0 \\
\hline Chimbarongo & 0 & 99 & 79 & 91 & 0 \\
\hline Chonchi & 0 & 0 & 0 & 0 & 677 \\
\hline Cisnes & 0 & 0 & 0 & 0 & 51 \\
\hline Cobquecura & 0 & 172 & 114 & 0 & 0 \\
\hline Cochamó & 0 & 0 & 0 & 0 & 0 \\
\hline Cochrane & 0 & 0 & 0 & 0 & 0 \\
\hline Codegua & 0 & 0 & 0 & 0 & 0 \\
\hline Coelemu & 0 & 204 & 0 & 0 & 0 \\
\hline Coihueco & 0 & 0 & 0 & 0 & 0 \\
\hline Coinco & 0 & 0 & 0 & 0 & 0 \\
\hline Colbún & 0 & 0 & 240 & 167 & 0 \\
\hline Colchane & 0 & 0 & 0 & 0 & 0 \\
\hline Colina & 151 & 57 & 41 & 0 & 0 \\
\hline Collipulli & 0 & 0 & 0 & 0 & 0 \\
\hline Coltauco & 157 & 0 & 0 & 256 & 0 \\
\hline Combarbalá & 0 & 0 & 0 & 109 & 0 \\
\hline Concepción & 2.823 & 1.770 & 1.396 & 0 & 0 \\
\hline Conchalí & 453 & 0 & 0 & 0 & 0 \\
\hline Concón & 0 & 0 & 0 & 0 & 0 \\
\hline Constitución & 0 & 621 & 134 & 82 & 38 \\
\hline Contulmo & 0 & 0 & 0 & 0 & 0 \\
\hline Copiapó & 1596 & 2221 & 82 & 0 & 0 \\
\hline Coquimbo & 2.104 & 1.714 & 217 & 173 & 450 \\
\hline Coronel & 1.424 & 1.510 & 0 & 347 & 174 \\
\hline Corral & 0 & 0 & 0 & 15 & 102 \\
\hline Coyhaique & 388 & 270 & 0 & 186 & 0 \\
\hline Cunco & 194 & 291 & 464 & 0 & 0 \\
\hline Curacautín & 50 & 326 & 34 & 0 & 0 \\
\hline Curacaví & 0 & 103 & 0 & 0 & 0 \\
\hline Curaco de Vélez & 0 & 0 & 0 & 0 & 0 \\
\hline Curanilahue & 420 & 804 & 0 & 161 & 0 \\
\hline Curarrehue & 0 & 0 & 0 & 0 & 0 \\
\hline Curepto & 0 & 0 & 0 & 32 & 0 \\
\hline Curicó & 787 & 1.919 & 215 & 524 & 0 \\
\hline Dalcahue & 0 & 0 & 0 & 0 & 0 \\
\hline Diego de Almagro & 204 & 361 & 0 & 0 & 0 \\
\hline Doñihue & 0 & 0 & 0 & 0 & 0 \\
\hline El Bosque & 1.060 & 830 & 121 & 0 & 0 \\
\hline El Carmen & 0 & 0 & 0 & 0 & 0 \\
\hline El Monte & 0 & 0 & 0 & 0 & 0 \\
\hline
\end{tabular}




\begin{tabular}{|c|c|c|c|c|c|}
\hline Comuna & Comercial & Industrial & Técnico & Agrícola & Marítimo \\
\hline El Quisco & 0 & 0 & 0 & 0 & 0 \\
\hline El Tabo & 0 & 0 & 0 & 0 & 0 \\
\hline Empedrado & 0 & 0 & 0 & 0 & 0 \\
\hline Ercilla & 0 & 0 & 0 & 0 & 0 \\
\hline Estación Central & 1.611 & 212 & 996 & 0 & 0 \\
\hline Florida & 125 & 0 & 0 & 0 & 0 \\
\hline Freire & 246 & 0 & 0 & 359 & 0 \\
\hline Freirina & 142 & 0 & 0 & 0 & 0 \\
\hline Fresia & 0 & 0 & 0 & 86 & 0 \\
\hline Frutillar & 169 & 408 & 157 & 0 & 0 \\
\hline Futaleufú & 0 & 0 & 0 & 0 & 0 \\
\hline Futrono & 0 & 0 & 139 & 163 & 0 \\
\hline Galvarino & 0 & 0 & 0 & 0 & 0 \\
\hline General Lagos & 0 & 0 & 0 & 0 & 0 \\
\hline Gorbea & 0 & 0 & 0 & 90 & 0 \\
\hline Graneros & 77 & 0 & 0 & 275 & 0 \\
\hline Guaitecas & 0 & 0 & 0 & 0 & 0 \\
\hline Hijuelas & 0 & 0 & 0 & 0 & 0 \\
\hline Hualaihué & 0 & 0 & 0 & 0 & 231 \\
\hline Hualañé & 0 & 0 & 0 & 0 & 0 \\
\hline Hualqui & 0 & 0 & 0 & 0 & 0 \\
\hline Huara & 0 & 0 & 0 & 119 & 0 \\
\hline Huasco & 230 & 0 & 0 & 0 & 86 \\
\hline Huechuraba & 1.065 & 736 & 79 & 0 & 0 \\
\hline Illapel & 0 & 792 & 0 & 0 & 0 \\
\hline Independencia & 2.822 & 3.213 & 2.703 & 0 & 0 \\
\hline Iquique & 3948 & 1.310 & 746 & 0 & 510 \\
\hline Isla de Maipo & 660 & 312 & 0 & 0 & 0 \\
\hline Isla de Pascua & 0 & 0 & 0 & 0 & 0 \\
\hline Juan Fernández & 0 & 0 & 0 & 0 & 0 \\
\hline La Cisterna & 9.059 & 5.161 & 2.978 & 0 & 0 \\
\hline La Cruz & 0 & 0 & 0 & 0 & 0 \\
\hline La Estrella & 0 & 0 & 0 & 0 & 0 \\
\hline La Florida & 3.286 & 755 & 357 & 0 & 0 \\
\hline La Granja & 1.087 & 163 & 305 & 0 & 0 \\
\hline La Higuera & 0 & 0 & 0 & 0 & 0 \\
\hline La Ligua & 285 & 0 & 20 & 160 & 0 \\
\hline La Pintana & 429 & 505 & 220 & 0 & 0 \\
\hline La Reina & 365 & 799 & 0 & 0 & 0 \\
\hline La Serena & 324 & 1.213 & 1.725 & 137 & 0 \\
\hline La Unión & 219 & 698 & 0 & 169 & 0 \\
\hline Lago Verde & 0 & 0 & 0 & 0 & 0 \\
\hline Lago Ranco & 0 & 0 & 0 & 0 & 0 \\
\hline Laguna Blanca & 0 & 0 & 0 & 0 & 0 \\
\hline
\end{tabular}




\begin{tabular}{|c|c|c|c|c|c|}
\hline Сотипа & Comercial & Industrial & Técnico & Agrícola & Marítimo \\
\hline Laja & 238 & 241 & 83 & 0 & 0 \\
\hline Lampa & 81 & 422 & 49 & 0 & 0 \\
\hline Lanco & 419 & 279 & 0 & 318 & 0 \\
\hline Las Cabras & 0 & 0 & 0 & 0 & 0 \\
\hline Las Condes & 379 & 134 & 0 & 0 & 0 \\
\hline Lautaro & 560 & 0 & 95 & 0 & 0 \\
\hline Lebu & 132 & 375 & 213 & 0 & 103 \\
\hline Licantén & 0 & 0 & 0 & 95 & 0 \\
\hline Limache & 0 & 0 & 97 & 0 & 0 \\
\hline Linares & 1.686 & 1.338 & 640 & 297 & 0 \\
\hline Litueche & 0 & 0 & 0 & 0 & 0 \\
\hline Llanquihue & 0 & 0 & 299 & 0 & 116 \\
\hline Llay Llay & 127 & 96 & 93 & 141 & 0 \\
\hline Lo Barnechea & 312 & 208 & 225 & 133 & 0 \\
\hline Lo Espejo & 308 & 198 & 42 & 0 & 0 \\
\hline Lo Prado & 362 & 46 & 64 & 0 & 0 \\
\hline Lolol & 0 & 0 & 0 & 69 & 0 \\
\hline Loncoche & 0 & 424 & 46 & 130 & 0 \\
\hline Longaví & 0 & 0 & 0 & 101 & 0 \\
\hline Lonquimay & 0 & 0 & 0 & 0 & 0 \\
\hline Los Álamos & 0 & 89 & 0 & 259 & 0 \\
\hline Los Andes & 955 & 1.035 & 589 & 0 & 0 \\
\hline Los Ángeles & 1.420 & 2.563 & 888 & 281 & 0 \\
\hline Los Lagos & 0 & 0 & 0 & 237 & 0 \\
\hline Los Muermos & 0 & 6 & 13 & 0 & 0 \\
\hline Los Sauces & 0 & 0 & 0 & 255 & 0 \\
\hline Los Vilos & 0 & 0 & 129 & 0 & 0 \\
\hline Lota & 991 & 1.380 & 54 & 0 & 0 \\
\hline Lumaco & 0 & 0 & 0 & 0 & 0 \\
\hline Machalí & 0 & 0 & 0 & 0 & 0 \\
\hline Macul & 1.534 & 415 & 394 & 0 & 0 \\
\hline Mafil & 0 & 0 & 0 & 144 & 0 \\
\hline Maipú & 1.880 & 2.647 & 0 & 0 & 0 \\
\hline Malloa & 0 & 0 & 0 & 0 & 0 \\
\hline Marchigüe & 0 & 0 & 0 & 0 & 0 \\
\hline María Elena & 61 & 246 & 0 & 0 & 0 \\
\hline María Pinto & 0 & 0 & 0 & 85 & 0 \\
\hline Mariquina & 0 & 0 & 0 & 190 & 109 \\
\hline Maule & 12 & 0 & 0 & 299 & 0 \\
\hline Maullín & 0 & 0 & 0 & 0 & 0 \\
\hline Mejillones & 0 & 45 & 16 & 0 & 0 \\
\hline Melipeuco & 0 & 0 & 0 & 0 & 0 \\
\hline Melipilla & 0 & 1.058 & 1.051 & 574 & 0 \\
\hline Molina & 294 & 0 & 386 & 546 & 0 \\
\hline
\end{tabular}




\begin{tabular}{|c|c|c|c|c|c|}
\hline Comuna & Comercial & Industrial & Técnico & Agrícola & Marítimo \\
\hline Monte Patria & 0 & 0 & 0 & 0 & 0 \\
\hline Mostazal & 0 & 106 & 0 & 0 & 0 \\
\hline Mulchén & 0 & 61 & 0 & 90 & 0 \\
\hline Nacimiento & 0 & 0 & 0 & 109 & 0 \\
\hline Nancagua & 157 & 0 & 0 & 137 & 0 \\
\hline Natales & 132 & 152 & 104 & 0 & 0 \\
\hline Navarino & 0 & 0 & 0 & 0 & 0 \\
\hline Navidad & 0 & 0 & 0 & 0 & 0 \\
\hline Negrete & 0 & 0 & 0 & 189 & 0 \\
\hline Ninhue & 0 & 0 & 0 & 0 & 0 \\
\hline Nogales & 0 & 346 & 334 & 182 & 0 \\
\hline Nueva Imperial & 816 & 408 & 375 & 400 & 0 \\
\hline Ñuñoa & 6.219 & 2.360 & 1.183 & 0 & 0 \\
\hline O'Higgins & 0 & 0 & 0 & 0 & 0 \\
\hline Olivar & 0 & 0 & 0 & 0 & 0 \\
\hline Ollagüe & 0 & 0 & 0 & 0 & 0 \\
\hline Olmué & 0 & 0 & 59 & 0 & 0 \\
\hline Osorno & 2.804 & 1.259 & 431 & 0 & 0 \\
\hline Ovalle & 218 & 957 & 771 & 318 & 0 \\
\hline Padre Hurtado & 500 & 257 & 109 & 0 & 0 \\
\hline Padre Las Casas & 122 & 1.017 & 67 & 0 & 0 \\
\hline Paihuano & 0 & 0 & 0 & 0 & 0 \\
\hline Paillaco & 0 & 125 & 0 & 191 & 0 \\
\hline Paine & 85 & 0 & 87 & 368 & 0 \\
\hline Palena & 0 & 0 & 0 & 0 & 0 \\
\hline Palmilla & 0 & 0 & 0 & 0 & 0 \\
\hline Panguipulli & 525 & 0 & 346 & 337 & 0 \\
\hline Panquehue & 0 & 0 & 0 & 0 & 0 \\
\hline Papudo & 0 & 0 & 0 & 0 & 0 \\
\hline Paredones & 0 & 0 & 0 & 0 & 0 \\
\hline Parral & 0 & 0 & 0 & 583 & 0 \\
\hline Pedro Aguirre Cerda & 1.475 & 251 & 639 & 0 & 0 \\
\hline Pelarco & 72 & 96 & 0 & 0 & 0 \\
\hline Pelluhue & 0 & 0 & 0 & 0 & 0 \\
\hline Pemuco & 0 & 0 & 0 & 155 & 0 \\
\hline Peñaflor & 1.091 & 108 & 152 & 0 & 0 \\
\hline Peñalolén & 1.513 & 1.083 & 818 & 0 & 0 \\
\hline Pencahue & 0 & 0 & 0 & 203 & 0 \\
\hline Penco & 0 & 0 & 0 & 0 & 120 \\
\hline Peralillo & 0 & 0 & 0 & 0 & 0 \\
\hline Perquenco & 0 & 0 & 0 & 0 & 0 \\
\hline Petorca & 0 & 0 & 0 & 106 & 0 \\
\hline Peumo & 0 & 0 & 101 & 134 & 0 \\
\hline Pica & 0 & 0 & 0 & 0 & 0 \\
\hline
\end{tabular}




\begin{tabular}{|c|c|c|c|c|c|}
\hline Comuna & Comercial & Industrial & Técnico & Agrícola & Marítimo \\
\hline Pichidegua & 0 & 0 & 0 & 0 & 0 \\
\hline Pichilemu & 0 & 0 & 0 & 91 & 0 \\
\hline Pinto & 0 & 116 & 179 & 0 & 0 \\
\hline Pirque & 0 & 0 & 0 & 238 & 0 \\
\hline Pitrufquén & 711 & 359 & 103 & 0 & 0 \\
\hline Placilla & 0 & 0 & 0 & 0 & 0 \\
\hline Portezuelo & 0 & 0 & 0 & 145 & 0 \\
\hline Porvenir & 24 & 21 & 0 & 0 & 0 \\
\hline Pozo Almonte & 215 & 222 & 12 & 0 & 0 \\
\hline Primavera & 0 & 0 & 0 & 0 & 0 \\
\hline Providencia & 1.700 & 0 & 400 & 0 & 0 \\
\hline Puchuncaví & 0 & 92 & 92 & 0 & 0 \\
\hline Pucón & 0 & 0 & 368 & 0 & 0 \\
\hline Pudahuel & 1.196 & 847 & 64 & 0 & 0 \\
\hline Puente Alto & 3.303 & 3.012 & 1.810 & 0 & 0 \\
\hline Puerto Montt & 1.646 & 1.237 & 714 & 0 & 138 \\
\hline Puerto Octay & 0 & 0 & 48 & 0 & 0 \\
\hline Puerto Varas & 278 & 73 & 142 & 0 & 0 \\
\hline Pumanque & 0 & 0 & 0 & 0 & 0 \\
\hline Punitaqui & 0 & 0 & 0 & 0 & 0 \\
\hline Punta Arenas & 1.225 & 1.407 & 651 & 0 & 0 \\
\hline Puqueldón & 0 & 0 & 0 & 0 & 0 \\
\hline Purén & 0 & 0 & 0 & 0 & 0 \\
\hline Purranque & 151 & 126 & 134 & 0 & 0 \\
\hline Putaendo & 0 & 0 & 0 & 0 & 0 \\
\hline Putre & 0 & 0 & 0 & 0 & 0 \\
\hline Puyehue & 113 & 0 & 93 & 119 & 99 \\
\hline Queilén & 0 & 0 & 0 & 0 & 0 \\
\hline Quellón & 0 & 0 & 0 & 0 & 59 \\
\hline Quemchi & 0 & 0 & 0 & 0 & 0 \\
\hline Quilaco & 0 & 0 & 0 & 0 & 0 \\
\hline Quilicura & 292 & 520 & 241 & 0 & 0 \\
\hline Quilleco & 0 & 0 & 0 & 0 & 0 \\
\hline Quillón & 0 & 0 & 0 & 0 & 0 \\
\hline Quillota & 1.168 & 624 & 0 & 152 & 0 \\
\hline Quilpué & 1.412 & 816 & 615 & 0 & 0 \\
\hline Quinchao & 0 & 0 & 106 & 55 & 99 \\
\hline Quinta de Tilcoco & 0 & 0 & 0 & 0 & 0 \\
\hline Quinta Normal & 3.237 & 4.181 & 1.449 & 0 & 0 \\
\hline Quintero & 0 & 137 & 0 & 0 & 152 \\
\hline Quirihue & 160 & 0 & 0 & 106 & 0 \\
\hline Rancagua & 2.836 & 1.699 & 954 & 0 & 0 \\
\hline Ranquil & 0 & 0 & 0 & 0 & 0 \\
\hline Rauco & 0 & 0 & 0 & 0 & 0 \\
\hline
\end{tabular}




\begin{tabular}{|c|c|c|c|c|c|}
\hline Сотипа & Comercial & Industrial & Técnico & Agrícola & Marítimo \\
\hline Recoleta & 2.425 & 2.024 & 386 & 0 & 0 \\
\hline Renaico & 0 & 0 & 0 & 148 & 0 \\
\hline Renca & 844 & 2.278 & 143 & 0 & 0 \\
\hline Rengo & 477 & 535 & 0 & 0 & 0 \\
\hline Requínoa & 73 & 56 & 0 & 0 & 0 \\
\hline Retiro & 95 & 58 & 0 & 77 & 0 \\
\hline Rinconada & 0 & 0 & 0 & 0 & 0 \\
\hline Río Bueno & 0 & 0 & 0 & 34 & 0 \\
\hline Río Claro & 0 & 207 & 0 & 0 & 0 \\
\hline Río Hurtado & 0 & 0 & 0 & 86 & 0 \\
\hline Río Ibáñez & 0 & 0 & 0 & 0 & 0 \\
\hline Río Negro & 0 & 141 & 0 & 234 & 0 \\
\hline Romeral & 0 & 0 & 0 & 226 & 0 \\
\hline Saavedra & 0 & 0 & 0 & 0 & 0 \\
\hline Sagrada Familia & 0 & 0 & 0 & 152 & 0 \\
\hline Salamanca & 0 & 0 & 0 & 0 & 0 \\
\hline San Antonio & 1.211 & 545 & 245 & 131 & 390 \\
\hline San Bernardo & 2.684 & 898 & 211 & 0 & 0 \\
\hline San Carlos & 801 & 567 & 278 & 151 & 0 \\
\hline San Clemente & 159 & 0 & 0 & 84 & 0 \\
\hline San Esteban & 125 & 0 & 0 & 0 & 0 \\
\hline San Fabián & 0 & 0 & 0 & 0 & 0 \\
\hline San Felipe & 785 & 870 & 146 & 273 & 0 \\
\hline San Fernando & 953 & 799 & 869 & 285 & 0 \\
\hline San Gregorio & 0 & 0 & 0 & 0 & 0 \\
\hline San Ignacio & 0 & 276 & 0 & 106 & 0 \\
\hline San Javier & 62 & 0 & 44 & 43 & 0 \\
\hline San Joaquín & 1.855 & 3.110 & 1.107 & 0 & 0 \\
\hline San José de Maipo & 0 & 176 & 0 & 0 & 0 \\
\hline San Juan de la Costa & 0 & 0 & 0 & 102 & 0 \\
\hline San Miguel & 6.432 & 2.719 & 2.398 & 0 & 0 \\
\hline San Nicolás & 0 & 0 & 0 & 0 & 0 \\
\hline San Pablo & 0 & 0 & 0 & 239 & 0 \\
\hline San Pedro & 0 & 0 & 0 & 0 & 0 \\
\hline San Pedro de Atacama & 0 & 0 & 0 & 92 & \\
\hline San Pedro de la Paz & 0 & 743 & 0 & 0 & 0 \\
\hline San Rafael & 0 & 0 & 0 & 0 & 0 \\
\hline San Ramón & 2.250 & 280 & 265 & 0 & 0 \\
\hline San Rosendo & 0 & 0 & 0 & 0 & 0 \\
\hline San Vicente & 0 & 0 & 0 & 270 & 0 \\
\hline Santa Bárbara & 0 & 0 & 0 & 0 & 0 \\
\hline Santa Cruz & 525 & 312 & 155 & 0 & 0 \\
\hline Santa Juana & 0 & 0 & 0 & 252 & 0 \\
\hline Santa María & 157 & 0 & 275 & 0 & 0 \\
\hline
\end{tabular}




\begin{tabular}{|c|c|c|c|c|c|}
\hline Comuna & Comercial & Industrial & Técnico & Agricola & Marítimo \\
\hline Santiago & 11.181 & 4.254 & 3.408 & 0 & 0 \\
\hline Santo Domingo & 0 & 0 & 0 & 0 & 0 \\
\hline Sierragorda & 0 & 0 & 0 & 0 & 0 \\
\hline Talagante & 1.225 & 437 & 1.154 & 241 & 0 \\
\hline Talca & 3.372 & 2.648 & 1.855 & 0 & 0 \\
\hline Talcahuano & 1.510 & 1.885 & 853 & 0 & 0 \\
\hline Taltal & 155 & 205 & 0 & 0 & 0 \\
\hline Temuco & 5.284 & 3.465 & 1.343 & 91 & 0 \\
\hline Teno & 170 & 0 & 0 & 126 & 0 \\
\hline Teodoro Schmidt & 0 & 0 & 0 & 99 & 0 \\
\hline Tierra Amarilla & 146 & 649 & 0 & 0 & 0 \\
\hline Til Til & 0 & 80 & 0 & 0 & 0 \\
\hline Timaukel & 0 & 0 & 0 & 0 & 0 \\
\hline Tirúa & 0 & 0 & 0 & 0 & 59 \\
\hline Tocopilla & 278 & 281 & 148 & 0 & 0 \\
\hline Toltén & 0 & 0 & 0 & 0 & 277 \\
\hline Tomé & 1.132 & 690 & 92 & 0 & 0 \\
\hline Torres del Paine & 0 & 0 & 0 & 0 & 0 \\
\hline Tortel & 0 & 0 & 0 & 0 & 0 \\
\hline Traiguén & 0 & 72 & 0 & 408 & 0 \\
\hline Trehuaco & 0 & 0 & 0 & 0 & 0 \\
\hline Tucapel & 0 & 0 & 0 & 97 & 0 \\
\hline Valdivia & 1.223 & 1.291 & 682 & 82 & 0 \\
\hline Vallenar & 591 & 973 & 0 & 183 & 0 \\
\hline Valparaíso & 2.240 & 2.404 & 2.064 & 0 & 1.612 \\
\hline Vichuquén & 0 & 0 & 0 & 0 & 0 \\
\hline Victoria & 321 & 577 & 108 & 0 & 0 \\
\hline Vicuña & 0 & 136 & 0 & 0 & 0 \\
\hline Vilcún & 121 & 476 & 47 & 371 & 0 \\
\hline Villa Alegre & 0 & 225 & 32 & 172 & 0 \\
\hline Villa Alemana & 634 & 0 & 116 & 0 & 238 \\
\hline Villarrica & 931 & 604 & 288 & 0 & 0 \\
\hline Viña del Mar & 2.597 & 1.914 & 228 & 0 & 0 \\
\hline Vitacura & 0 & 0 & 0 & 0 & 0 \\
\hline Yerbas Buenas & 0 & 44 & 31 & 406 & 0 \\
\hline Yumbel & 0 & 0 & 0 & 0 & 0 \\
\hline Yungay & 0 & 0 & 0 & 0 & 0 \\
\hline Zapallar & 0 & 0 & 0 & 0 & 0 \\
\hline
\end{tabular}

Fuente: Mineduc. 


\section{REFERENCIAS}

Arellano, \& M. Braun (1999). "Rentabilidad de la educación formal en Chile”. Cuadernos de Economía 107, Instituto de Economía. Pontificia Universidad Católica de Chile.

Bassi, M., \& S. Urzúa (2010). "Educación en Chile: El desafío está en la calidad”. IDB Publications 37998, Inter-American Development Bank.

BID (Banco Interamericano de Desarrollo) (2008). "Encuesta sobre trayectorias y habilidades (ETH). Chile". Washington D.C.: BID.

Bishop, J., \& F. Mane (2004). "The impacts of career-technical education on high school labor market success". Economics of Education Review, 23(4), 381-402.

Bucarey, A., \& S. Urzúa (2013). "The economic return to vocational high schools in Chile". Mimeo, University of Maryland.

Card, D. (1999). "The causal effect of education on earnings". En O. Ashenfelter, \& D. Card (ed.), Handbook of Labor Economics. Vol. 3 de Handbook of Labor Economics, cap. 30, pp. 1801-1863. Elsevier. (2001). "Estimating the return to schooling: Progress on some persistent econometric problems", Econometrica, 69(5), 1127-60.

CNED (2010). Acuerdo Número 093/2010. Consejo Nacional de Educación (CNED).

De Iruarrizaga, F. (2009). "Dos miradas a la educación media en Chile". Tesis magister en economía, Instituto de Economía. Pontificia Universidad Católica de Chile.

Farías, M., \& M. P. Sevilla (2012). "Efectividad de la enseñanza media técnico profesional en la persistencia y rendimiento en la educación técnica superior". Documento de trabajo, Mineduc.

Gustman, A., \& T. Steinmeier (1982). "The relation between vocational training in high school and economic outcomes". Industrial and Labor Relations Review, 36(1), 73-87.

Heckman, J. J., \& B. E. Honore (1990). "The empirical content of the roy model". Econometrica, 58(5), 1121-49.

Heckman, J. J., \& P. A. LaFontaine (2006). "Bias-corrected estimates of GED Returns". Journal of Labor Economics, 24(3), 661-700.

Heckman, J. J., S. Urzúa, \& E. Vytlacil (2006). "Understanding instrumental variables in models with essential heterogeneity". The Review of Economics and Statistics, 88(3), 389-432.

Malamud, O., \& C. Pop-Eleches (2010). "General Education versus Vocational Training: Evidence from an Economy in Transition". Review of Economics and Statistics, 92(1), 43-60.

Meer, J. (2007). "Evidence on the returns to secondary vocational education". Economics of Education Review, 26(5), 559-573.

Meghir, C., \& S. Rivkin (2011). Econometric Methods for Research in Education. Vol. 3 del Handbook of the Economics of Education, Elsevier. cap. 1, pp. 1-87. 
Mineduc (2009). "Bases para una política de formación técnico-profesional en Chile". Informe de la Comisión para el Estudio de la Formación Técnico-Profesional en Chile.

Moenjak, T., \& C. Worswick (2003). "Vocational education in Thailand: A study of choice and returns". Economics of Education Review, 22(1), 99-107.

Neuman, S., \& A. Ziderman (1999). "Vocational education in Israel: Wage effects of the voced-occupation match". Journal of Human Resources, 34(2), 407-420.

- (2003). "Can vocational education improve the wages of minorities and disadvantaged groups? The case of Israel". Economics of Education Review, 22(4), 421-432.

Newhouse, D., \& D. Suryadarma (2011). "The value of vocational education: High school type and labor market outcomes in Indonesia". World Bank Economic Review, 25(2), 296-322.

OECD (2012). Education at a Glance 2012 OECD Indicators: OECD Indicators, Education at a Glance, OECD Publishing.

Reyes, L., J. Rodríguez, \& S. Urzúa (2012). "The economic returns to postsecondary degrees in Chile". Discussion paper, borrador.

Sapelli, C. (2009). "Los retornos a la educación en Chile: Estimaciones por corte transversal y por cohortes". Documentos de Trabajo 349, Instituto de Economía, Pontificia Universidad Católica de Chile.

Staiger, D., \& J. H. Stock (1997). "Instrumental Variables Regression with Weak Instruments". Econometrica, 65(3), 557-586.

Trost, R. P., \& L.-F. Lee (1984). "Technical training and earnings: A polychotomous choice model with selectivity". The Review of Economics and Statistics, 66(1), 151-56.

Urzúa, S. (2012). "La rentabilidad de la educación superior en Chile". Estudios Públicos, N 125 (verano 2012). 